\title{
Bending strength predictions of cross-laminated timber plates subjected to concentrated loading using 3D finite-element-based limit analysis approaches
}

\author{
Mingjing Li ${ }^{* 1}$, Josef Füssl ${ }^{1}$, Markus Lukacevic ${ }^{1}$, Chris M. Martin ${ }^{2}$, and Josef \\ Eberhardsteiner ${ }^{1}$ \\ ${ }^{1}$ Vienna University of Technology, Karlsplatz 13/202, Vienna 1040, Austria \\ ${ }^{2}$ University of Oxford, Parks Road, Oxford OX1 3PJ, UK
}

February 27, 2019

\begin{abstract}
Cross-laminated timber (CLT) is an innovative wood product with increasing utilisations. It is well-known that, the orthotropic and inhomogeneous strength properties of wooden boards have a strong influence on the load bearing capacity of CLT plates, especially when the complex wood fibre distribution due to randomly occurring knots is considered. Thus, high safety factors are used in current standards and a generally-accepted numerical tool for the strength prediction of CLT plates is still not available. In this paper, we combine recent advances in 3D numerical limit analysis and a knot reconstruction algorithm, where not only the bending strength of CLT plates under concentrated loading is estimated using the numerical approach, but also the scatter of strength properties resulting from the material's inhomogeneities is investigated using the stochastic approach. For the latter, data collected during the grading process of wooden boards is condensed into so-called strength profiles for single wooden boards. The limit analysis approach then allows a time efficient simulation of a large number of randomly assembled CLT plates. The comparison of the resulting strength predictions to experiments shows good agreement with respect to both the mean load bearing capacity and the statistical scatter of strength.
\end{abstract}

\section{Introduction}

Wood is undergoing a revival and recapturing market shares from other building materials, reflected in an enormous growth rate of the overall volume consumption. Beside its excellent mechanical and physical properties, wood is well-known as an environmentally sustainable material with a pleasant appearance, and thus, particularly suitable for residential buildings, office buildings, school buildings, and related buildings. The main reasons for this upswing are continuous improvements in building codes throughout Europe on the one hand and the development and application of innovative woodbased products on the other hand.

One of the most aspiring products is cross-laminated timber (CLT), which is a plate-like composite consisting of an odd number of layers with wooden boards placed side-by-side in each layer and stacked crosswise. The basic idea of CLT was developed in Austria and Germany in the early 1990s. From the early 2000s, this innovative wood product has driven global interest and intensive research efforts have been carried out starting from Central Europe and spreading over the world. The utilisation

\footnotetext{
*E-mail address: mingjing.li@tuwien.ac.at (M. Li).
} 
of CLT panels in constructions has sharply increased during the last decade, particularly in mid-rise and high-rise buildings in both Europe and North America. This achievement can be attributed to its outstanding properties regarding in- and out-of-plane bearing capacity, degree of prefabrication, seismic performance, fire resistance, sound insulation, as well as to its environmentally sustainable characteristics $[7,23]$.

Despite the increasing demand, the current utilisation of wood-based products with respect to their mechanical properties is far away from their real potential [21], because dimensioning practice and many existing design rules are still based on empirical observations / background.

Regarding CLT, as reviewed by Hochreiner et al. [26] and Brandner et al. [7], a large number of research has been performed on a wide range of topics, but particularly for strength, those research is either limited to the evaluation of failure modes on the surface of CLT elements or only considers the mechanical behaviour within the elastic regime. For this reason, no design method for CLT has been universally accepted at this moment, which is a drawback for the ongoing CLT standardisation [7]. For example, the Eurocode 5 [18] design concept for CLT elements is based on the first violation of either bending or rolling shear strength, and the mechanical behaviour beyond the elastic regime is not considered, leaving room for a better utilisation of the material. This could be achieved by efficient and reliable computational methods, providing more information about the mechanical behaviour, especially with respect to failure mechanisms and realistic collapse loads. The main difficulty for the implementation of such computational methods for CLT is inherited from its raw material wood, which, as a typical naturally-grown material, exhibits a very complex hierarchical structure and a significant variety in macroscopic mechanical properties.

However, the application of computational methods to determine the strength of wooden elements is a rapidly growing area of research and many contributions can be found in the near past. The first group of approaches uses the mean stress concept, where averaged stresses over a finite small area are assumed to indicate failure instead of the direct description of failure mechanism $[1,64,81-83]$. More sophisticated approaches are based on so-called multi-surface plasticity/failure criteria, where stress states leading to cracks are determined by orthotropic yield surfaces [59,78]. An extension of this approach is the implementation of cohesive zone models to simulate the crack propagation in wood [79, 80]. The assumption of a homogeneous fibre orientation is required for such methods, which is normally unrealistic for wood, especially in the presence of knots and other defects. Thus, in recent years, two of the authors $[51,53,54]$ have established the basis for a crack initiation and propagation criterion in the framework of the eXtended Finite Element Method (XFEM). Since microscopic failure mechanisms have significant influence on the macroscopic failure behaviour of wood, in these works, the concepts of plastic failure criteria as well as crack initiation criteria were applied to several length scales to finally obtain a multi-surface failure criterion for wood at the macroscale. With such implementations, a realistic numerical description of failure mechanisms within wooden boards seems to be possible in the near future.

For CLT, because of its laminated structure with wooden lamellas exhibiting variable mechanical properties aligned in an orthogonal manner, numerical modelling becomes even more difficult. Saavedra Flores et. al introduced a promising numerical approach based on a multiscale concept using different homogenisation schemes predicting the stiffness behaviour $[28,74,75]$ and the rolling shear failure behaviour [73]. However, their work mainly focuses on stiffness properties and only specific failure mechanism, rolling shear failure, has been studied, and each layer of the CLT plate is defined as homogeneous with no variations between wooden boards. To the authors' knowledge, a numerical approach regarding failure behaviour of CLT with non-homogeneous strength properties of lamellas hasn't been proposed yet.

Additionally, as stated by Hochreiner et al. [27], the strength properties of wooden boards have a strong influence on the load bearing capacity of CLT, which has rarely been studied in previous research, neither experimentally nor numerically. The variety in mechanical properties between wooden 
boards can be partly attributed to the varying clear wood properties, e.g. density and moisture content, and more importantly to the presence of knots and fibre deviations around them. As stated by Johansson [30], in most destructive bending or tension tests, failure is caused either by knots themselves or by cut fibres due to local slope of grain in their vicinities. Lukacevic and Füssl [50] presented a physically-based structural failure criterion, where it was assumed that in wooden boards with knots, global failure can be related to a stress transfer mechanism, which is identifiable by evaluating averaged stress fields in the vicinity of knots. Complemented by a knot reconstruction algorithm [36, 52], mechanical properties can be predicted for wooden board sections containing knots by using so-called indicating properties [52], which are originally used during the strength grading process of such boards. By dividing wooden boards into a reasonable number of clear wood and knot sections, which are able to represent the distribution of mechanical properties, the global performance of wood-based products can be estimated based on a stochastic approach, as successfully implemented for stiffness estimates of glued laminated timber (GLT) beams by Kandler et al. [33-35], or it can even be applied to the optimization of the assembly of such beams by using metaheuristic algorithms [69]. Since the main focus of this work is on strength properties, a similar stochastic approach can be applied to estimate the load bearing capacity of CLT by using above-mentioned strength profiles of wooden boards. For this purpose, comprehensive numerical calculations are required, which must be able to consider highly orthotropic and non-linear material behaviour.

Recently, the numerical limit analysis has been implemented by Li et al. [46] for strength estimates of wood, providing promising results at different length scales and exhibiting higher time efficiency and a simpler solution procedure compared to conventional finite element based approaches [22]. This numerical approach has been widely used as a powerful prediction tool for load bearing capacities and failure mechanisms of various structures, e.g. reinforced-concrete structures [12,47,48,72], composite laminates [43, 49, 70,71] and masonry structures [65-67], in which calculations exhibit high time efficiency and promising performance. Considering these features, the numerical limit analysis is expected to be a suitable alternative tool to estimate the load bearing capacity, especially of CLT, where less brittle and more ductile failure mechanisms are observed in general.

Originally, the objective of limit analysis was the determination of the load bearing capacity of structures exhibiting elastoplastic material response. At collapse, the capacity of structures to store any additional external work as recoverable energy is lost and limit analysis concentrates on the critical energy dissipation rate at structural failure. The problem may be stated as follows: Find the kinematically admissible velocity field, which minimises the external energy over the set of all statically admissible stress fields, which maximise the internal dissipated energy according to Ciria et al. [11]. Unfortunately, the so-obtained saddle-point problem can be solved exactly only for simple geometric and loading situations, and for simple material behaviour. For more complex situations, the plastic flow compatibility in the static lower bound principle and the plastic admissibility in the kinematic upper bound principle may be relaxed, providing lower and upper bounds for the load bearing capacity (effective strength) of structures.

The first complete formulations of both lower bound and upper bound theorems were established by Drucker et al. $[14,15]$ as well as Hill [25], and an early implementation of the finite element method and the optimisation theory into limit analysis was done by [58] for the lower bound problem, and by [2] and [60] for the upper bound problem. In these works, linear three-noded triangular elements were used for discretisation and the underlying optimisation problem could be solved by linear programming, provided that the used failure criteria were linearised.

Thanks to the rapid evolution of computer technology and the development in mathematical programming, more attention has been drawn to numerical limit analysis formulations within past decades. Notable contributions with respect to the non-linear programming have been given by [39, 55-57], enabling non-linear failure criteria being formulated in their native form. However, local smoothing of yield surfaces with singularities, like the Tresca or Mohr-Coulomb critera, were necessary. 
In general, the efficiency of the numerical limit analysis method significantly depends on the algorithms for solving the associated optimisation problem. In later studies, as proposed by Makrodimopoulos and Martin [62,63], Ciria and Peraire [10], and Krabbenhøft et al. [40], the second-order cone programming (SOCP) has been proven to be an excellent alternative method, with sufficient robustness and efficiency to solve large-scale optimisation problems of limit analysis. This optimisation algorithm allows the use of quadratic cone-shaped yield functions in their native nonlinear form without local smoothing and avoids the common difficulties of general non-linear programming in handling non-differentiability at the apex point. Thus, within this work, the SOCP is employed for the proposed numerical limit analysis formulations.

Although isotropic strength behaviour is usually considered in limit analysis formulations, the use of orthotropic yield functions has been discussed by Capsoni et al. [8,9] and $\mathrm{Li}$ [42], and the formulation of the Tsai-Wu yield function, which is commonly used for wood [13, 16, 50, 54,59], as second-order cone (SOC) constraints in numerical limit analysis formulations has been recently presented by the authors $[22,46]$.

The main objective of this work has been to develop and apply 3D numerical limit analysis formulations to determine the bearing capacity of CLT plates (under bending) and, additionally, considering the strength property variations within wooden lamellas according to several grading classes, which in reality are caused by knots and fibre deviations. In Section 2, the complete 3D numerical limit analysis formulations are introduced for both the lower bound and the upper bound approaches. As a validation, these formulations are applied to CLT plate bending tests of three different grading classes to obtain strength estimates in Section 3.1. Then, this numerical method is combined with a stochastic approach for the CLT plate bending capacity using strength profiles in Section 3.2. Finally, a brief conclusion is given in Section 4.

\section{Numerical limit analysis formulations}

Classical limit analysis is based on plastic bounding theorems, requiring the assumptions of ideal plasticity and a linearised geometrical framework, but not necessarily the assumption of rigidity, as sometimes stated otherwise. However, applying limit analysis only to elastic perfectly plastic materials would probably waste the potential of this method, as similarly stated in [76]. For any material with the potential to activate plastic resistance along a possible failure surface, limit analysis might lead to appropriate estimates for its collapse load/effective strength.

The main objective is to determine the collapse load multiplier $\beta$ and corresponding failure modes for a given load situation under certain boundary conditions. The procedure can be briefly summarised as follows: Finding the statically admissible stress field maximising the external load and the kinematically admissible velocity field minimising the internal plastic energy dissipation delivers a lower bound $\beta^{L B}$ and an upper bound $\beta^{U B}$ of the exact collapse load multiplier $\beta^{*}$, respectively. The computational error can be easily estimated as

$$
\operatorname{err}(\%)=\left(\beta^{U B}-\beta^{L B}\right) \cdot 100 /\left(\beta^{U B}+\beta^{L B}\right)
$$

In this section, both 3D lower bound and upper bound formulations are introduced in discretised form. More detailed derivations and expressions can be found in related publications from the authors $[44,46]$.

\subsection{Lower bound formulation}

The lower bound optimisation problem is looking for the maximum external load while the stress field satisfies internal equilibrium, the static boundary conditions, and the plastic failure criteria. It can 
be formulated as

$$
\begin{array}{lll}
\beta^{L B}=\max & \beta \\
\text { s.t. } & \operatorname{div} \boldsymbol{\sigma}=\mathbf{g} & \text { in } \Omega \\
& \boldsymbol{\sigma} \cdot \mathbf{n}=\beta \mathbf{t} & \text { on } \Gamma_{b c} \\
& f(\boldsymbol{\sigma}) \leq 0 & \text { in } \Omega
\end{array}
$$

where $\Omega$ refers to the volume of the structure with $\Gamma=\partial \Omega$ as the boundary surface, $\boldsymbol{\sigma} \in \mathfrak{R}^{3 \times 3}$ represents the Cauchy stress field, div is the divergence operator for stress tensors, $\mathbf{g} \in \mathfrak{R}^{3}$ is the body force field, $\mathbf{n} \in \mathfrak{R}^{3}$ is the outward normal vector on the surface part $\Gamma_{b c} \subset \Gamma$ where a boundary surface traction field $\mathbf{t} \in \mathfrak{R}^{3}$ is prescribed, and $f(\boldsymbol{\sigma}) \leq 0$ indicates the plastic yield function.

For the discretisation of this lower bound formulation, tetrahedron elements with 4 internal stress evaluation nodes (at the 4 vertices) are used. The stress field $\boldsymbol{\sigma}$ is interpolated within each tetrahedron element using linear shape functions $N_{\sigma}^{e l, i}$, and the nodal stress vector is expressed in Voigt notation $\mathbf{q}_{\sigma}^{e l, i} \in \mathfrak{R}^{6}(\forall e l \in\{1, \cdots, L E\}$ and $i \in\{1, \cdots, 4\})$ with $L E$ as the total number of elements in the lower bound problem. In the discretised structure, the traction field $\mathbf{t}$ is enforced to be continuous between elements, but jumps in the stress field $\boldsymbol{\sigma}$ are allowed across element interfaces. Thus, all elements have their own internal stress evaluation nodes and, at adjacent nodes, stress evaluation nodes from different elements are allowed to share the same position.

\subsubsection{Equilibrium within elements}

The first constraint in Eq. (2) represents the internal equilibrium within each element $e l$, which can be formulated in discretised form as

$$
\sum_{i=1}^{4} \mathbf{L}_{\sigma} N_{\sigma}^{e l, i} \mathbf{q}_{\sigma}^{e l, i}=\mathbf{q}_{g}^{e l}, \quad \forall e \in\{1, \cdots, L E\}
$$

where $\mathbf{L}_{\sigma} \in \mathfrak{R}^{3 \times 6}$ represents the divergence operator for the stress vector in Voigt notation and $\mathbf{q}_{g}^{e l} \in \mathfrak{R}^{3}$ denotes the elemental body force vector.

\subsubsection{Equilibrium between elements}

To enforce continuity of the traction field between elements the following additional constraint is applied:

$$
\mathbf{L}_{t}^{d e} \mathbf{q}_{\sigma}^{d e, i}-\mathbf{L}_{t}^{d e} \mathbf{q}_{\sigma}^{d e, i^{\prime}}=\mathbf{0}, \quad \forall d e \in\{1, \cdots, L D E\} \text { and } i \in\{1, \cdots, 3\}
$$

where $\mathbf{q}_{\sigma}^{d e, i} \in \mathfrak{R}^{6}$ is the nodal stress vector for each pair of the 3-noded triangular interfaces de, with $i$ and $i^{\prime}$ indicating the nodal pairs. $L D E$ is the total number of element interfaces in the lower bound problem and $\mathbf{L}_{t}^{d e} \in \mathfrak{R}^{3 \times 6}$ represents the stress-traction operator based on the normal vector $\mathbf{n}^{\text {de }} \in \mathfrak{R}^{3}$ for each element interface $d e$, pointing from the node $i$ to the node $i^{\prime}$.

\subsubsection{Static boundary conditions}

The traction field on the boundary surface part $\Gamma_{b c}$ is prescribed through the second constraint in the optimisation problem Eq. (2). In the discretised form this constraint is applied to the triangular boundary surfaces, denoted as be, on $\Gamma_{b c}$, reading

$$
\mathbf{L}_{t}^{b e} \mathbf{q}_{\sigma}^{b e, i}=\beta \mathbf{q}_{t}^{b e, i}, \quad \forall b e \in\{1, \cdots, L B E\} \text { and } i \in\{1, \cdots, 3\}
$$

where $L B E$ is the total number of triangular surfaces on $\Gamma_{b c}, \mathbf{q}_{\sigma}^{b e, i}$ is the nodal stress vector of the triangular surface, $\mathbf{q}_{t}^{b e, i}$ is the surface traction vector, and $\mathbf{L}_{t}^{b e}$ is the stress-traction operator for each boundary element based on the corresponding outward normal vector $\mathbf{n}^{b e}$ on $\Gamma_{b c}^{b e} \subset \Gamma_{b c}$. 


\subsubsection{Plastic failure criteria}

In the lower bound formulation, the plastic failure criteria are applied at each stress evaluation node to determine the plastic failure state. In this work, the yield function is expressed in a general form for the Tsai-Wu failure criterion as

$$
\begin{array}{r}
\mathbf{q}_{\sigma, l o c}^{e l, i}=\mathbf{R}_{\sigma}^{e l} \mathbf{q}_{\sigma}^{e l, i} \\
\begin{aligned}
& f\left(\mathbf{q}_{\sigma, l o c}^{e l, i}, k_{\sigma}^{e l}\right)=\mathbf{q}_{\sigma, l o c}^{e l, i}{ }^{\top} \mathbf{P}_{\sigma}^{e l} \mathbf{q}_{\sigma, l o c}^{e l, i}+\mathbf{F}_{\sigma}^{e l \top} \mathbf{q}_{\sigma, l o c}^{e l, i}-1 \leq 0 \\
&, \quad \forall e l \in\{1, \cdots, L E\} \text { and } i \in\{1, \cdots, 4\}
\end{aligned}
\end{array}
$$

where $\mathbf{q}_{\sigma, l o c}^{e l, i} \in \mathfrak{R}^{6}$ is the nodal local stress vector with respect to the principal material orientation in the element $e l, \mathbf{R}_{\sigma}^{e l} \in \mathfrak{R}^{6 \times 6}$ is the transformation matrix for stress vectors from the global coordinate basis $(x-y-z)$ into the material local coordinate basis $\left(x^{\prime}-y^{\prime}-z^{\prime}\right)_{\text {mat }}^{e l}$ for each element. The matrices $\mathbf{P}_{\sigma}^{e l} \in \mathfrak{R}^{6 \times 6}$ and $\mathbf{F}_{\sigma}^{e l} \in \mathfrak{R}^{6}$ are determined by the type of yield function and the material strength parameters applied to the element $e l$.

As shown in Eq. (2), the nonlinearity of the lower bound optimisation problem is attributed to the nonlinear yield function $f(\boldsymbol{\sigma}) \leq 0$. Thus, this nonlinear optimisation problem can be solved efficiently using SOCP as long as the yield function Eq. (6) can be formulated as a SOC constraint, reading

$$
\begin{aligned}
& \mathbf{s}_{\sigma}^{e l, i}=\mathbf{a}_{\sigma}^{e l}+\mathbf{B}_{\sigma}^{e l} \mathbf{R}_{\sigma}^{e l} \mathbf{q}_{\sigma}^{e l, i} \\
& \mathbf{s}_{\sigma}^{e l, i} \in \mathscr{C}
\end{aligned}, \quad \forall e l \in\{1, \cdots, L E\} \text { and } i \in\{1, \cdots, 4\}
$$

where $\mathscr{C}$ refers to the quadratic cone, $\mathbf{s}_{\sigma}^{e l, i} \in \mathfrak{R}^{8}$ represents the SOC expression of the yield function. $\mathbf{a}_{\sigma}^{e l} \in \mathfrak{R}^{8}$ and $\mathbf{B}_{\sigma}^{e l} \in \mathfrak{R}^{8 \times 6}$ are determined from $\mathbf{P}_{\sigma}^{e l}$ and $\mathbf{F}_{\sigma}^{e l}$.

\subsubsection{Discretised lower bound optimisation problem}

Assembling above constraints, the lower bound optimisation problem Eq. (2) can be written in discretised form as

$$
\begin{array}{ll}
\beta^{L B}=\max & \beta \\
\text { s.t. } & \mathbf{A}_{\sigma}^{\text {mat }} \mathbf{q}_{\sigma}=\mathbf{q}_{g} \\
& \mathbf{A}_{t}^{d i s} \mathbf{q}_{\sigma}=\mathbf{0} \\
& \mathbf{A}_{t}^{b c} \mathbf{q}_{\sigma}=\beta \mathbf{q}_{t} \\
& \mathbf{s}_{\sigma}=\mathbf{a}_{\sigma}+\mathbf{B}_{\sigma} \mathbf{R}_{\sigma} \mathbf{q}_{\sigma} \\
& \mathbf{s}_{\sigma} \in \mathscr{C}
\end{array}
$$

where $\mathbf{q}_{\sigma} \in \mathfrak{R}^{24 L E}$ contains all nodal stress vectors throughout the whole structure, $\mathbf{q}_{g} \in \mathfrak{R}^{3 L E}$ is the assembled body force vector for all elements, $\mathbf{q}_{t} \in \mathfrak{R}^{9 L B E}$ is the assembled boundary traction vector, $\mathbf{s}_{\sigma} \in \mathfrak{R}^{32 L E}$ is the assembled vector for SOC expressions of yield functions at all stress evaluation nodes, $\mathbf{a}_{\sigma} \in \mathfrak{R}^{32 L E}$ and $\mathbf{B}_{\sigma} \in \mathfrak{R}^{32 L E \times 24 L E}$ are assembled matrices containing material strength parameters from Eq. (7). $\mathbf{A}_{\sigma}^{\text {mat }} \in \mathfrak{R}^{3 L E \times 24 L E}, \mathbf{A}_{t}^{d i s} \in \mathfrak{R}^{9 L D E \times 24 L E}, \mathbf{A}_{t}^{b c} \in \mathfrak{R}^{9 L B E \times 24 L E}$ and $\mathbf{R}_{\sigma} \in \mathfrak{R}^{24 L E \times 24 L E}$ are assembled throughout the whole structure from Eqs. (3), (4), (5) and (7), respectively.

\subsection{Upper bound formulation}

Based on the theorem, the objective of upper bound calculations is looking for the failure mechanism for which the corresponding kinematically admissible velocity field $\dot{\mathbf{u}} \in \mathfrak{R}^{3}$ leads to the lowest internal energy dissipation rate $W_{i n t}$.

In this work, as is introduced in [44,45], velocity jumps $\Delta \dot{\mathbf{u}} \in \mathfrak{R}^{3}$ are allowed only across prescribed discontinuities $\Gamma_{d i s}$, consisting of a surface $\Gamma_{d i s}^{+}$with the related velocity field $\dot{\mathbf{u}}_{d i s}^{+}$and a surface $\Gamma_{d i s}^{-}$ 
with the related velocity field $\dot{\mathbf{u}}_{d i s}^{-}$. Such velocity jumps $\Delta \dot{\mathbf{u}}=\left(\dot{\mathbf{u}}_{d i s}^{+}-\dot{\mathbf{u}}_{d i s}^{-}\right)$represent additional degrees of freedom for the plastic flow, locally at $\Gamma_{d i s}$. Then, the internal energy dissipation rate $W_{\text {int }}$ is composed of the plastic energy dissipation rate in the continuum body $\Omega$ and the dissipation rate due to interface failure across the prescribed discontinuities $\Gamma_{d i s}$, giving

$$
W_{\text {int }}=\int_{\Omega} d_{p}^{m a t}(\dot{\boldsymbol{\varepsilon}}) \mathrm{d} V+\int_{\Gamma_{\text {dis }}} d_{p}^{d i s}(\dot{\boldsymbol{\mu}}) \mathrm{d} A
$$

with the plastic dissipation functions as

$$
\begin{array}{ll}
d_{p}^{\text {mat }}=\sup _{\boldsymbol{\sigma} \in \mathcal{F}} \boldsymbol{\sigma}^{\top} \dot{\boldsymbol{\varepsilon}}, \quad \mathcal{F}=\{\boldsymbol{\sigma} \mid f(\boldsymbol{\sigma}) \leq 0\} & \text { in } \Omega \\
d_{p}^{\text {dis }}=\sup _{\mathbf{t} \in \mathcal{D}} \mathbf{t}^{\top} \dot{\boldsymbol{\mu}}, \quad \mathcal{D}=\{\mathbf{t} \mid f(\mathbf{t}) \leq 0\} & \text { on } \Gamma_{\text {dis }}
\end{array}
$$

where $\dot{\boldsymbol{\mu}} \in \mathfrak{R}^{3}$ is the local plastic flow related to the velocity-jump field $\Delta \dot{\mathbf{u}}$ at $\Gamma_{d i s}, f(\boldsymbol{\sigma}) \leq 0$ and $f(\mathbf{t}) \leq 0$ are the stress-based yield function for $\Omega$ and the traction-based yield function for $\Gamma_{d i s}$, respectively.

Then, the primal upper bound optimisation problem can be formulated as

$$
\begin{array}{lll}
\min & W_{\text {int }} & \\
\text { s.t. } & \dot{\boldsymbol{\varepsilon}}=\mathbf{L}_{\dot{u}} \dot{\mathbf{u}} & \text { in } \Omega \\
& \dot{\mathbf{u}}=\dot{\mathbf{u}}_{b c} & \text { on } \Gamma_{b c} \\
& \dot{\boldsymbol{\varepsilon}}=\dot{\lambda}_{\sigma} \partial f(\boldsymbol{\sigma}) / \partial \boldsymbol{\sigma} & \text { in } \Omega \\
& \dot{\boldsymbol{\mu}}=\dot{\lambda}_{t} \partial f(\mathbf{t}) / \partial \mathbf{t} & \text { on } \Gamma_{d i s}
\end{array}
$$

in which the four constraints are related to compatibility, kinematic boundary conditions, and associated plastic flow both in $\Omega$ as well as at $\Gamma_{d i s}$. In the first constraint, $\mathbf{L}_{\dot{u}} \in \mathfrak{R}^{6 \times 3}$ denotes the linear compatibility operator mapping the velocity field $\dot{\mathbf{u}}$ onto the strain-rate field $\dot{\varepsilon}$. In the second constraint, $\dot{\mathbf{u}}_{b c}$ refers to the prescribed velocity boundary conditions at the boundary surface part $\Gamma_{b c} \subset \Gamma$. In the last two constraints, $\dot{\lambda}_{\sigma}$ and $\dot{\lambda}_{t}$ are the plastic multipliers determining the magnitude of plastic flow within the continuum material $\Omega$ and at the prescribed velocity discontinuity $\Gamma_{d i s}$, respectively.

The upper bound optimisation problem in Eq. (11) can also be formulated in a, mathematically equivalent, dual form, in which the objective function becomes the external work rate $W_{\text {ext }}$, reading

$$
W_{e x t}=\int_{\Omega} \dot{\mathbf{u}}^{\top} \mathbf{g}_{0} \mathrm{~d} V+\int_{\Gamma_{b c}} \dot{\mathbf{u}}^{\top} \beta \mathbf{t}_{b c} \mathrm{~d} A
$$

consisting of the work done by a constant body force field $\mathbf{g}_{0}$ in $\Omega$ and the boundary surface traction field $\mathbf{t}_{b c}$ on $\Gamma_{b c}$. The complete dual upper bound problem can then be formulated as follows

$$
\begin{array}{lll}
\max & W_{\text {ext }} & \\
\text { s.t. } & \int_{\Omega} \dot{\boldsymbol{\varepsilon}}^{\top} \boldsymbol{\sigma} \mathrm{d} V+\int_{\Gamma_{d i s}} \dot{\boldsymbol{\mu}}^{\top} \mathbf{t} \mathrm{d} A=\int_{\Omega} \dot{\mathbf{u}}^{\top} \mathbf{g}_{0} \mathrm{~d} V+\int_{\Gamma_{b c}} \dot{\mathbf{u}}^{\top} \beta \mathbf{t} \\
& f(\boldsymbol{\sigma}) \leq 0 & \text { in } \Omega \\
& f(\mathbf{t}) \leq 0 & \text { in } \Omega \\
& \text { on } \Gamma_{\text {dis }}
\end{array}
$$

where the first constraint refers to the weak form of equilibrium, related to Eq. (9) and Eq. (12), the second and the third constraints represent the stress-based and traction-based yield functions for the continuum material $\Omega$ and the velocity discontinuities $\Gamma_{d i s}$, respectively. Note that, in this work, the body force is not considered that $\mathbf{g}_{0}=\mathbf{0}$ and an additional constraint is applied that $\int_{\Gamma_{b c}} \dot{\mathbf{u}}^{\top} \mathbf{t}_{b c} \mathrm{~d} A=1$, and, thus, the objective function of the optimisation problem Eq. 13 can be reformulated as $W_{\text {int }}=\beta$. 
In the following, only this dual optimisation problem is discretised and solved, but complete expressions of discretised primal and dual upper bound formulations can be found in $[44,45]$. For the discretisation linear strain tetrahedron elements are used with 10 velocity evaluation nodes (4 vertice and 6 middle nodes) and 4 strain-rate evaluation nodes (at the 4 vertices). The velocity field $\dot{\mathbf{u}}$ is approximated using 3D quadratic shape functions $N_{\dot{u}}^{e l, i}$ for interpolation between the nodal velocities $\mathbf{q}_{\dot{u}}^{e l, i} \in \mathfrak{R}^{3}(\forall e l \in\{1, \cdots, U E\}$ and $i \in\{1, \cdots, 10\})$ with $U E$ as the total number of elements; the strain-rate field $\dot{\varepsilon}$ is approximated using 3D linear shape functions $N_{\dot{\varepsilon}}^{e l, j}$ for the interpolation between the nodal strain-rate vectors $\mathbf{q}_{\dot{\varepsilon}}^{e l, i} \in \mathfrak{R}^{6}(\forall e l \in\{1, \cdots, U E\}$ and $j \in\{1, \cdots, 4\})$.

On the boundary surface part $\Gamma_{b c}$, each boundary surface element be contains 6 velocity evaluation nodes (3 vertice and 3 middle nodes). The velocity field $\dot{\mathbf{u}}$ is approximated using $2 \mathrm{D}$ quadratic shape functions $\bar{N}_{\dot{u}}^{b e, i}$ for interpolation between the nodal velocity vectors $\mathbf{q}_{\dot{u}}^{b e, i} \in \mathfrak{R}^{3}(\forall b e \in\{1, \cdots, U B E\}$ and $i \in$ $\{1, \cdots, 6\})$ within $\Gamma_{b c}^{b e} \subset \Gamma_{b c}$, where $U B E$ is the total number of 6-noded boundary elements on $\Gamma_{b c}$.

In the discretised upper bound formulation, the strain-rate field $\dot{\varepsilon}$ varies linearly within each element and is allowed to be discontinuous between elements; velocity jumps $\Delta \dot{\mathbf{u}}$ are allowed only across prescribed velocity discontinuities $\Gamma_{d i s}$.

At $\Gamma_{d i s}$, each triangular discontinuity element de contains 6 velocity evaluation nodes (3 vertice and 3 middle nodes), where 2D quadratic shape functions $\bar{N}_{\dot{u}}^{d e, i}$ are used to interpolate between 6 pairs of nodal velocity vectors $\mathbf{q}_{\dot{u}}^{d e+, j}$ and $\mathbf{q}_{\dot{u}}^{d e-, j}(\forall d e \in\{1, \cdots, U D E\}$ and $i \in\{1, \cdots, 6\})$ with $U D E$ as the total number of discontinuity elements on $\Gamma_{d i s}$. To guarantee consistence of plastic flow throughout the whole structure, it is straightforward to enforce the localised plastic flow $\dot{\boldsymbol{\mu}}$ to be linear at each discontinuity element $\Gamma_{\text {dis }}^{\text {de }} \subset \Gamma_{d e}$, where 3 internal evaluation nodes (at the 3 vertices) are used to interpolate the localised plastic flow $\dot{\boldsymbol{\mu}}$ using 2D linear shape functions $\bar{N}_{\dot{\mu}}^{d e, i}$ to interpolate between the nodal plastic flow vectors $\mathbf{q}_{\dot{\mu}}^{d e, j} \in \mathfrak{R}^{3}(\forall d e \in\{1, \cdots, U D E\}$ and $j \in\{1, \cdots, 3\})$.

\subsubsection{Weak equilibrium}

Strictly speaking, as stated by Makrodimopoulos [61], the upper bound problem is based on the kinematic theorem, in which only the velocity field $\dot{\mathbf{u}}$ and the strain-rate field $\dot{\boldsymbol{\varepsilon}}$ are to be considered. Thus, the forces appearing in the upper bound formulations, partly due to exploiting duality, not necessarily represent physically accurate interpretable quantities. They can rather be interpreted as stress-like quantities $\hat{\boldsymbol{\sigma}}^{e l}$ and traction-like quantities $\hat{\mathbf{t}}^{d e}$, defined as follows

$$
\begin{aligned}
\hat{\boldsymbol{\sigma}}^{e l} & =\sum_{i=1}^{4} \frac{\int_{\Omega^{e l}} N_{\dot{\varepsilon}}^{e l, i} \boldsymbol{\sigma} \mathrm{d} V}{\int_{\Omega^{e l}} N_{\dot{\varepsilon}}^{e l, i} \mathrm{~d} V}, \quad \forall e l \in\{1, \cdots, U E\} \\
\hat{\mathbf{t}}^{d e} & =\sum_{j=1}^{3} \frac{\int_{\Gamma_{d e}^{d e}} \bar{N}_{\dot{\mu}}^{d e, j} \mathbf{t d} A}{\int_{\Gamma_{d i s}^{d e}} \bar{N}_{\dot{\mu}}^{d e, j} \mathrm{~d} A}, \quad \forall d e \in\{1, \cdots, U D E\}
\end{aligned}
$$

where both evaluation nodes and shape functions are shared with the strain-rate field $\dot{\varepsilon}$ in each solid element $\Omega^{e l} \subset \Omega$ and the localised plastic flow $\dot{\boldsymbol{\mu}}$ at each discontinuity element $\Gamma_{\text {dis }}^{\text {de }}$, respectively.

The internal energy dissipation rate Eq. (9), consisting of the plastic dissipation in both the material as well as at the interface, can then be discretised as follows

$$
\begin{aligned}
W_{\text {int }} & =\sum_{e l=1}^{U E} \int_{\Omega^{e l}} \dot{\boldsymbol{\varepsilon}}^{\top} \hat{\boldsymbol{\sigma}}^{e l} \mathrm{~d} V+\sum_{d e=1}^{U D E} \int_{\Gamma_{d i s}^{d e}} \dot{\boldsymbol{\mu}}^{\top} \hat{\mathbf{t}}^{d e} \mathrm{~d} A \\
& =\sum_{e l=1}^{U E} \sum_{i=1}^{4} \sum_{j=1}^{4} \mathbf{q}_{\dot{\varepsilon}}^{e l, i \top} \hat{\mathbf{q}}_{\sigma}^{e l, j} \int_{\Omega^{e l}} N_{\dot{\varepsilon}}^{e l, i} N_{\dot{\varepsilon}}^{e l, j} \mathrm{~d} V+\sum_{d e=1}^{U D E} \sum_{k=1}^{3} \sum_{l=1}^{3} \mathbf{q}_{\dot{\mu}}^{d e, k \top} \hat{\mathbf{q}}_{t}^{d e, l} \int_{\Gamma_{d i s}^{d e}} \bar{N}_{\dot{\mu}}^{d e, k} \bar{N}_{\dot{\mu}}^{d e, l} \mathrm{~d} V
\end{aligned}
$$


where $\hat{\mathbf{q}}_{\sigma}^{e l, i} \in \mathfrak{R}^{6}$ denotes the nodal stress-like vector in $\Omega^{e l}$ and $\hat{\mathbf{q}}_{t}^{d e, i} \in \mathfrak{R}^{3}$ the nodal traction-like vector at $\Gamma_{\text {dis }}^{\text {de }}$.

The compatibility constraint for each solid element in $\Omega^{e l}$ in discretised form reads

$$
\sum_{i=1}^{4} N_{\dot{\varepsilon}}^{e l, i} \mathbf{q}_{\dot{\varepsilon}}^{e l, i}=\mathbf{L}_{\dot{u}} \sum_{j=1}^{10} N_{\dot{u}}^{e l, j} \mathbf{q}_{\dot{u}}^{e l, j}, \quad \forall e l \in\{1, \cdots, U E\}
$$

indicating the relation between the nodal strain-rate vectors $\mathbf{q}_{\dot{\varepsilon}}^{e l, i}$ and the nodal velocity vectors $\mathbf{q}_{\dot{u}}^{e l, j}$; the compatibility constraint for each discontinuity element at $\Gamma_{d i s}^{d e}$ reads

$$
\sum_{i=1}^{3} \mathbf{q}_{\dot{\mu}}^{d e, i}=\sum_{j=1}^{6} \mathbf{L}_{\dot{\mu}} \mathbf{q}_{\dot{u}}^{d e, j}, \quad \forall d e \in\{1, \cdots, U D E\}
$$

where,

$$
\mathbf{L}_{\dot{\mu}}=\left(\begin{array}{ll}
\mathbf{I} & \mathbf{I}
\end{array}\right) \in \mathfrak{R}^{3 \times 6}
$$

indicating the relation between the nodal localised plastic flow vector $\mathbf{q}_{\dot{\mu}}^{d e, i}$ and the vectors for the nodal velocity pairs $\mathbf{q}_{\dot{u}}^{d e, j}=\left(\mathbf{q}_{\dot{u}}^{d e+, j}, \mathbf{q}_{\dot{u}}^{d e-, j}\right)^{\top} \in \mathfrak{R}^{6 \times 3}$.

Inserting Eqs. (16) and (17) into Eq. (15), the internal energy dissipation rate can be reformulated as,

$$
W_{i n t}=\sum_{e l=1}^{U E} \sum_{i=1}^{10} \sum_{j=1}^{4}\left(\mathbf{L}_{\dot{u}} \mathbf{q}_{\dot{u}}^{e l, i}\right)^{\top} \hat{\mathbf{q}}_{\sigma}^{e l, j} \int_{\Omega^{e l}} N_{\dot{u}}^{e l, i} N_{\dot{\varepsilon}}^{e l, j} \mathrm{~d} V+\sum_{d e=1}^{U D E} \sum_{k=1}^{6} \sum_{l=1}^{3}\left(\mathbf{L}_{\dot{\mu}} \mathbf{q}_{\dot{u}}^{d e, k}\right)^{\top} \hat{\mathbf{q}}_{t}^{d e, l} \int_{\Gamma_{d i s}^{d e}} \bar{N}_{\dot{u}}^{d e, k} \bar{N}_{\dot{\mu}}^{d e, l} \mathrm{~d} V
$$

In this work, the body force is not considered, giving $\mathbf{g}=\mathbf{0}$. According to Eq. (12), the discretised form of the external work rate can then be expressed as

$$
\begin{aligned}
W_{e x t} & =\sum_{b e=1}^{U B E} \int_{\Gamma_{b c}^{b e}} \dot{\mathbf{u}}^{\top} \beta \mathbf{t}_{b c} \mathrm{~d} A \\
& =\sum_{b e=1}^{U B E} \sum_{i=1}^{6} \sum_{j=1}^{n_{t}} \beta \mathbf{q}_{\dot{u}}^{b e, i \top} \mathbf{q}_{t_{b c}}^{b e, j} \int_{\Gamma_{b c}^{b e}} \bar{N}_{\dot{u}}^{b e, i} \bar{N}_{t_{b c}}^{b e, j} \mathrm{~d} A
\end{aligned}
$$

where the nodal traction vectors $\mathbf{q}_{t b c}^{b e, i}$ are prescribed at $n_{t}$ evaluation nodes at each boundary surface element on $\Gamma_{b c}^{b e}$, interpolated by the $2 \mathrm{D}$ shape functions $\bar{N}_{t_{b c}}^{b e, j}$ for the traction field $\mathbf{t}_{b c}$. Note that, since the boundary traction field $\mathbf{t}_{b c}$ is prescribed directly to each evaluation node on $\Gamma_{p}, \mathbf{q}_{t_{b c}}^{b e, i}$ can be expressed directly in the exact form.

\subsubsection{Plastic yield function}

The dual upper bound problem Eq. (13) consists of the stress-based yield functions $f(\boldsymbol{\sigma}) \leq 0$ for the continuum material $\Omega$ and the traction-based yield functions $f(\mathbf{t}) \leq 0$ for the prescribed velocity discontinuities $\Gamma_{d i s}$. In order to guarantee consistent strength behaviour throughout the whole structure, an algorithm has been introduced by the authors to project the stress-based yield function $f(\boldsymbol{\sigma}) \leq 0$ onto a locally equivalent traction-based yield function $f(\mathbf{t}) \leq 0$ with respect to $\Gamma_{\text {dis }}[44,45]$.

Since both the stress-like quantities $\hat{\boldsymbol{\sigma}}^{e l}$ and the traction-like quantities $\hat{\mathbf{t}}^{d e}$ are interpolated linearly within corresponding elements, it is sufficient to evaluate yield functions only at vertices of the 
elements. As introduced in Eq. (7), both yield functions can be formulated in SOC form, giving

$$
\begin{aligned}
& \hat{\mathbf{s}}_{\sigma}^{e l, i}=\hat{\mathbf{a}}_{\sigma}^{e l}+\hat{\mathbf{B}}_{\sigma}^{e l} \mathbf{R}_{\sigma}^{e l} \hat{\mathbf{q}}_{\sigma}^{e l, i}, \quad \forall e l \in\{1, \cdots, U E\} \text { and } i \in\{1, \cdots, 4\} \\
& \hat{\mathbf{s}}_{\sigma}^{e l, i} \in \mathscr{C} \\
& \hat{\mathbf{s}}_{t}^{d e, j}=\hat{\mathbf{a}}_{t}^{d e}+\hat{\mathbf{B}}_{t}^{d e} \mathbf{R}_{t}^{d e} \hat{\mathbf{q}}_{t}^{d e, j}, \quad \forall d e \in\{1, \cdots, U D E\} \text { and } j \in\{1, \cdots, 3\} \\
& \hat{\mathbf{s}}_{t}^{d e, j} \in \mathscr{C}
\end{aligned}
$$

where $\mathbf{R}_{t}^{d e}$ represents the transformation matrix for traction vectors from the global coordinate basis $(x-y-z)$ to the local coordinate basis $\left(x^{\prime}-y^{\prime}-z^{\prime}\right)_{d i s}^{d e}$ for velocity discontinuities, $\hat{\mathbf{s}}_{\sigma}^{e l, i} \in \mathfrak{R}^{8}$ and $\hat{\mathbf{s}}_{t}^{d, i} \in \mathfrak{R}^{5}$ are the SOC expressions of the yield functions, $\hat{\mathbf{a}}_{\sigma}^{e l, i} \in \mathfrak{R}^{8}, \hat{\mathbf{B}}_{\sigma}^{e l, i} \in \mathfrak{R}^{8 \times 6}$ and $\hat{\mathbf{a}}_{t}^{d, i} \in \mathfrak{R}^{5}$, $\hat{\mathbf{B}}_{t}^{d, i} \in \mathfrak{R}^{5 \times 3}$ contain material strength parameters. In Eq. (21), the yield functions are formulated in terms of the weighted averaged expressions according to Eq. (14). As stated by Makrodimopoulos [61], only when the material strength properties are constant within each element, the weighted averaged quantities equal the exact nodal quantities and all hat symbols can be ignored in Eqs. (15), (14) and (21).

\subsubsection{Assembly}

Finally, the discretised formulation of the dual upper bound optimisation problem, Eq. (13), can be formulated as

$$
\begin{array}{ll}
\max & W_{e x t} \\
\text { s.t. } & \mathbf{A}_{U B}^{\text {mat }} \hat{\mathbf{q}}_{\sigma}^{\text {mat }}+\mathbf{A}_{U B}^{\text {dis }} \hat{\mathbf{q}}_{t}^{\text {dis }}=\beta \mathbf{A}_{U B}^{b c} \mathbf{q}_{t}^{b c} \\
& \hat{\mathbf{s}}_{\sigma}^{\text {mat }}=\hat{\mathbf{a}}_{\sigma}^{\text {mat }}+\hat{\mathbf{B}}_{\sigma}^{\text {mat }} \mathbf{R}_{\sigma}^{\text {mat }} \hat{\mathbf{q}}_{\sigma}^{\text {mat }} \\
& \hat{\mathbf{s}}_{\sigma}^{\text {mat }} \in \mathscr{C} \\
& \hat{\mathbf{s}}_{t}^{\text {dis }}=\hat{\mathbf{a}}_{t}^{\text {dis }}+\hat{\mathbf{B}}_{t}^{\text {dis }} \mathbf{R}_{t}^{\text {dis }} \hat{\mathbf{q}}_{t}^{\text {dis }} \\
& \hat{\mathbf{s}}_{t}^{\text {dis }} \in \mathscr{C}
\end{array}
$$

where $\mathbf{A}_{U B}^{m a t} \in \mathfrak{R}^{3 U N \times 24 U E}$ and $\mathbf{A}_{U B}^{\text {dis }} \in \mathfrak{R}^{3 U N \times 9 U D}$ are assembled from Eq. (19) with $U N$ as the total number of velocity evaluation nodes; $\mathbf{q}_{t}^{b c} \in \mathfrak{R}^{3 U N}$ and $\mathbf{A}_{U B}^{b c} \in \mathfrak{R}^{3 U N \times 3 n_{t} U B C}$ are assembled from Eq. (20); $\hat{\mathbf{s}}_{\sigma}^{\text {mat }} \in \mathfrak{R}^{32 U E}, \hat{\mathbf{a}}_{\sigma}^{\text {mat }} \in \mathfrak{R}^{32 U E}, \hat{\mathbf{B}}_{\sigma}^{\text {mat }} \in \mathfrak{R}^{32 U E \times 24 U E}, \mathbf{R}_{\sigma}^{\text {mat }} \in \mathfrak{R}^{24 U E \times 24 U E}$ and $\hat{\mathbf{s}}_{t}^{\text {dis }} \in \mathfrak{R}^{15 U D}$, $\hat{\mathbf{a}}_{t}^{\text {dis }} \in \mathfrak{R}^{15 U D}, \hat{\mathbf{B}}_{t}^{\text {dis }} \in \mathfrak{R}^{15 U D \times 9 U D}, \mathbf{R}_{t}^{\text {dis }} \in \mathfrak{R}^{9 U D \times 9 U D}$ are assembled from Eq. (21); $\hat{\mathbf{q}}_{\sigma}^{\text {mat }} \in \mathfrak{R}^{24 U E}$ and $\hat{\mathbf{q}}_{t}^{d i s} \in \mathfrak{R}^{9 U D}$ are the assembly of weighted average vectors for nodal stress vectors in $\Omega$ and nodal traction vectors on $\Gamma_{d i s}$, respectively.

Note that, the assembled velocity vector $\mathbf{q}_{\dot{u}} \in \mathfrak{R}^{2 U N}$ has been eliminated in both side of the first constraint in Eq. (22) since nodal velocity vectors exist in each term of the discretised internal dissipation rate Eq. (19) and external work rate Eq. (20).

\section{Application to CLT plates}

In this section, the proposed numerical limit analysis formulations are applied to estimate the bending bearing capacities of CLT plates. The CLT plates are implemented as layer laminated structures consisting of wooden boards aligned parallely in each layer and perpendicularly over adjacent layers. For each wooden board, anisotropic failure behaviour is considered using the Tsai-Wu failure criterion. All CLT plates are discretised by above-mentioned tetrahedron elements. Strength parameters are prescribed for each element, by which, the distribution of strength properties along wooden boards can be considered easily by prescribed strength profiles. 
According to experimental observations from Hochreiner et al. [26,27], the dominant failure mechanism across wooden boards is rolling shear failure in wood next to the glued interfaces since the rolling shear strength of wood is significantly weaker than the glue shear strength. Thus, in this paper, both board-to-board and layer-to-layer interfaces are considered as perfectly-glued and failure is considered only within wooden boards.

\subsection{CLT plate bending test}

The first task is applying the numerical method to estimate the CLT plate bending capacity for several grading classes and comparing the numerical results with experimental observations [27]. In the experimental setup, CLT plates were assembled using wooden boards from 3 different strength classes (C18, C24, and C35) according to the European code EN338, where each plate consisted of wooden boards from the same strength class. Each CLT plate exhibits a 3 layered structure with dimensions of $1500 \times 1500 \times 57 \mathrm{~mm}$ and each layer consisted of 15 parallelly-aligned wooden boards with dimensions of $1500 \times 100 \times 19 \mathrm{~mm}$.

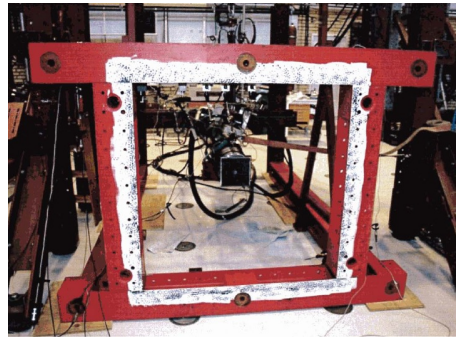

(a)

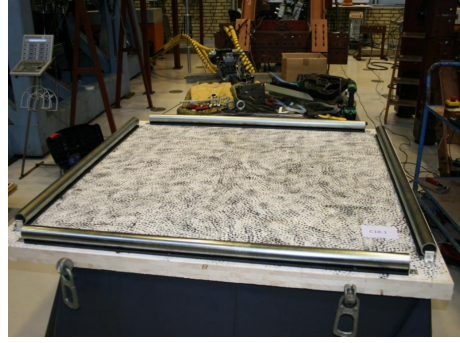

(b)

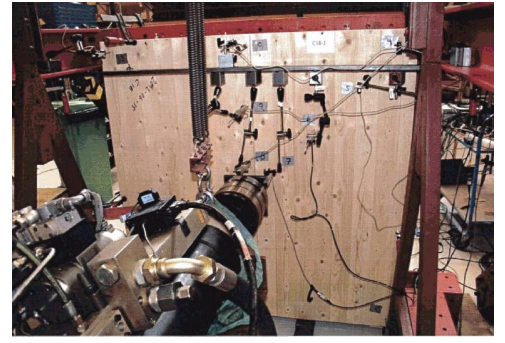

(c)

Figure 1: Illustration of the experimental set-up: (a) vertically oriented supporting steel frame with horizontal loading; (b) four pre-assembled tubes as the supporting structure on loaded front surface of CLT plates; (c) hydraulic punch device as the concentrated loading on the center of loaded back surface [27].

As shown in Figure 1a, the CLT plate was mounted on a vertically oriented steel frame and a concentrated loading was applied horizontally. Against the loading direction, the plate was supported by 4 pre-assembled tubes on the loaded front surface as line constraints, shown in Figure 1b. The loading was applied by a hydraulic punch device on the centre of the loaded surface with a square loaded area of $200 \times 200 \mathrm{~mm}$, shown in Figure 1c.

In this way, 8 CLT specimens were tested for each of the 3 strength classes (C18, C24, and C35) and the influence of the wooden board strength properties on the CLT plate load bearing capacities were discussed [27].

Dimensions of the numerical models for the CLT plates, shown in Figure 2a, are defined according to the experimental setup. As shown in Figure 2b, the loading condition is applied as a uniform vertical velocity field at all nodes in the loaded region on the top surface, illustrated as the blue square in Figure 2a, and boundary conditions are applied by fixing the vertical degree of freedom for all nodes on the line constraints on the bottom, illustrated as the blue dashed lines in Figure 2a. Each CLT plate is discretised using approximately 40,000 tetrahedral elements (see Figure 2c for an example). An orthotropic Tsai-Wu yield function is applied to each element with respect to the local coordinate basis determined from the alignment of the corresponding wooden board.

As a validation, the numerical approaches are expected to provide reliable estimates on the load bearing capacities of CLT plates compared to experimental results. For this purpose, the differences in strength properties between the strength classes have to be considered, and it is straightforward to determine strength parameters according to EN338. Note that these code strength values represent 


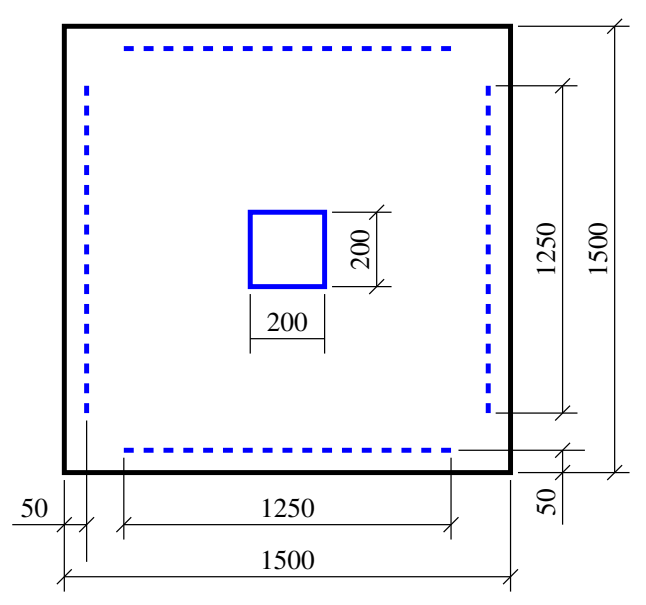

(a)

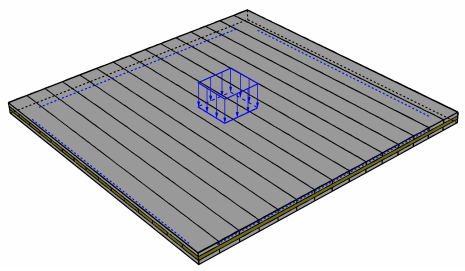

(b)

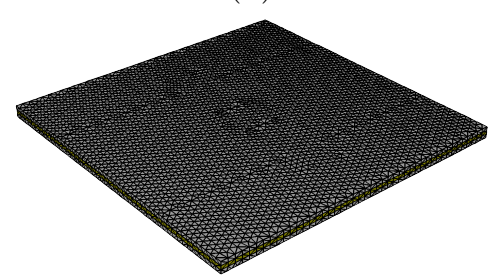

(c)

Figure 2: Numerical implementation of the CLT plate bending test as: (a) dimensions of the numerical model according to the experiment set-up with the blue square as loading region and the blue dash line as line constrints; (b) assembly of the wooden boards and the prescribed boundary conditions; (c) example of model discretisation.

\begin{tabular}{|c|c|c|c|c|c|}
\hline \multirow{2}{*}{ strength class } & \multicolumn{5}{|c|}{ strength properties $\left(\mathrm{N} / \mathrm{mm}^{2}\right)$} \\
\cline { 2 - 6 } & $f_{t, 0}$ & $f_{t, 90}$ & $f_{c, 0}$ & $f_{c, 90}$ & $f_{\tau}$ \\
\hline C18 & 15.70 & 0.53 & 19.90 & 2.59 & 4.55 \\
\hline C24 & 19.98 & 0.53 & 23.20 & 2.94 & 5.36 \\
\hline C35 & 29.67 & 0.53 & 27.62 & 3.29 & 5.36 \\
\hline
\end{tabular}

Table 1: Mean strength values for different strength classes of wooden boards calculated from characteristic strength values in European Code EN338, including tension parallel $f_{t, 0}$, tension perpendicular $f_{t, 90}$, compression parallel $f_{c, 0}$, compression perpendicular $f_{c, 90}$ and Shear $f_{\tau}$.

the characteristic 5\%-fractile-strengths, but for the comparison with experimental results it is more meaningful to use mean strength values as shown in Table (1), which are determined from characteristic values as introduced by Jenkel et al. [29]. Then, the strength parameters for the Tsai-Wu yield function Eq. (6) are commonly determined as

$$
\begin{array}{ll}
F_{\sigma, 1}=\frac{1}{f_{t, 0}}-\frac{1}{f_{c, 0}}, & F_{\sigma, 2}=F_{\sigma, 3}=\frac{1}{f_{t, 90}}-\frac{1}{f_{c, 90}}, \\
P_{\sigma, 11}=\frac{1}{f_{t, 0} f_{c, 0}}, & P_{\sigma, 22}=P_{\sigma, 33}=\frac{1}{f_{t, 90} f_{c, 90}}, \\
P_{\sigma, 55}=\frac{1}{\left(f_{\tau} / 3\right)^{2}}, \text { and } & P_{\sigma, 44}=P_{\sigma, 66}=\frac{1}{f_{\tau}{ }^{2}},
\end{array}
$$

where the material is considered as transversely isotropic, such that the strength values are identical in radial $(R)$ and tangential direction $(T)$, and the rolling shear strength is considered as one-third of the shear strength value in Table (1). As a result of using strength values obtained from codes, in the perpendicular-to-grain directions, the tensile strength $f_{t, 90}$ is much smaller than the compressive strength $f_{c, 90}$. In addition, the definition of the failure criterion with the components of Eq. (23) leads to an ellipsoidal failure surface, where the intersections of the ellipsoid with the coordinate axis conform with the chosen strength values. But the transformation of the failure surface towards the 
perpendicular-to-grain compressive stress region leads to unrealistically high maximum strength values in the parallel-to-grain direction, which are much larger than the desired, chosen strength values, in this direction. Thus, rotations and shrinkages are introduced to the Tsai-Wu failure surfaces by introducing interaction parameters $\left(P_{\sigma, 12}, P_{\sigma, 23}\right.$ and $\left.P_{\sigma, 13}\right)$ and adjusting normal strength parameters $\left(F_{\sigma, 1}, F_{\sigma, 2}, F_{\sigma, 3}, P_{\sigma, 11}, P_{\sigma, 22}\right.$ and $\left.P_{\sigma, 33}\right)$ based on following optimisation algorithm,

$$
\min \left(\sigma_{L L}^{\max }-f_{t, 0}\right)+\left(\sigma_{L L}^{\min }-f_{c, 0}\right)+\left(\sigma_{R R}^{\max }-f_{t, 90}\right)+\left(\sigma_{R R}^{\min }-f_{c, 90}\right)+\left(\sigma_{T T}^{\max }-f_{t, 90}\right)+\left(\sigma_{T T}^{\min }-f_{c, 90}\right)
$$

where $\sigma_{L L}^{\max }, \sigma_{R R}^{\max }, \sigma_{T T}^{\max }$ and $\sigma_{L L}^{\min }, \sigma_{R R}^{\min }, \sigma_{T T}^{\min }$ are the maximum and minimum strength values of the Tsai-Wu failure surfaces in the $L, R$ and $T$ directions, respectively. This leads to the following strength parameters in Eq. 25 for the three considered strength classes.

$$
\begin{aligned}
& \mathbf{P}_{\sigma}=\left(\begin{array}{cccccc}
0.0089 & -0.0096 & -0.0049 & 0.0 & 0.0 & 0.0 \\
-0.0096 & 0.2080 & -0.1629 & 0.0 & 0.0 & 0.0 \\
-0.0049 & -0.1629 & 0.1460 & 0.0 & 0.0 & 0.0 \\
0.0 & 0.0 & 0.0 & 0.0483 & 0.0 & 0.0 \\
0.0 & 0.0 & 0.0 & 0.0 & 0.4344 & 0.0 \\
0.0 & 0.0 & 0.0 & 0.0 & 0.0 & 0.0483
\end{array}\right) \quad \mathbf{F}_{\sigma}=\left(\begin{array}{c}
0.0033 \\
0.0980 \\
0.0980 \\
0.0 \\
0.0 \\
0.0
\end{array}\right) \text { for C18 } \\
& \mathbf{P}_{\sigma}=\left(\begin{array}{cccccc}
0.0039 & -0.0036 & -0.0015 & 0.0 & 0.0 & 0.0 \\
-0.0036 & 0.1093 & -0.0867 & 0.0 & 0.0 & 0.0 \\
-0.0015 & -0.0867 & 0.0741 & 0.0 & 0.0 & 0.0 \\
0.0 & 0.0 & 0.0 & 0.0349 & 0.0 & 0.0 \\
0.0 & 0.0 & 0.0 & 0.0 & 0.3136 & 0.0 \\
0.0 & 0.0 & 0.0 & 0.0 & 0.0 & 0.0349
\end{array}\right) \quad \mathbf{F}_{\sigma}=\left(\begin{array}{c}
0.0014 \\
0.0534 \\
0.0534 \\
0.0 \\
0.0 \\
0.0
\end{array}\right) \text { for C24 } \\
& \mathbf{P}_{\sigma}=\left(\begin{array}{cccccc}
0.0016 & -0.0011 & -0.0004 & 0.0 & 0.0 & 0.0 \\
-0.0011 & 0.0507 & -0.0404 & 0.0 & 0.0 & 0.0 \\
-0.0004 & -0.0404 & 0.0334 & 0.0 & 0.0 & 0.0 \\
0.0 & 0.0 & 0.0 & 0.0349 & 0.0 & 0.0 \\
0.0 & 0.0 & 0.0 & 0.0 & 0.3136 & 0.0 \\
0.0 & 0.0 & 0.0 & 0.0 & 0.0 & 0.0349
\end{array}\right) \quad \mathbf{F}_{\sigma}=\left(\begin{array}{c}
0.0005 \\
0.0254 \\
0.0254 \\
0.0 \\
0.0 \\
0.0
\end{array}\right) \text { for C35 }
\end{aligned}
$$

With the proposed numerical limit analysis approach, the bending bearing capacities of CLT plates are estimated for all three strength classes and the results are compared to the experimental data. In the experiments, 8 CLT plates were tested for each strength class, and the collapse load from each test is plotted in Figure 3 with black crosses. Due to the uncertainty of the wooden board strength properties, the collapse loads from experiments for each strength class are distributed with certain scatter as discussed by Hochreiner et al. [27]. In numerical calculations, where constant strength parameters are used for wooden boards from the same strength class, the numerical results are plotted as blue lines in Figure 3, indicating one collapse load for each strength class. Small bound gaps between lower bound and upper bound results are observed for each strength configuration, which indicates small computational errors, which can be determined according to Eq. (1) as 1.39\% for $\mathrm{C} 18,1.86 \%$ for $\mathrm{C} 24$ and $0.83 \%$ for C35, suggesting an accurate approximation of the exact solution. Regarding the collapse load, the numerical results (blue lines) are close to the mean of the scatter of the experimental data points (black crosses) for the strength class C35, but the numerical calculations tend to overestimate the collapse load for the other 2 strength classes, C18 and C24. Since limit 


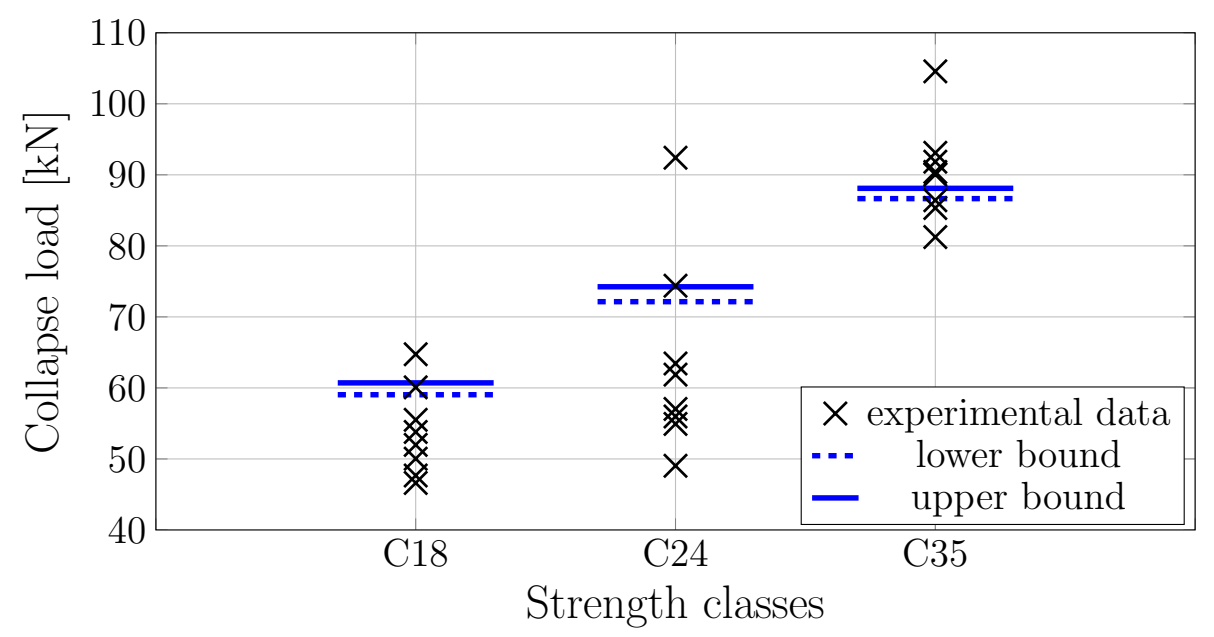

Figure 3: Numerical and experimental ultimate bending capacity of CLT plates using wooden boards from different strength classes.

analysis considers only ductile failure, this agrees well with the experimental observation in [27] that, the structural collapse in CLT plates of C35 is dominated by ductile rolling shear failure but brittle failure mechanisms also play an important role in the weaker CLT plates of C18 and C24.

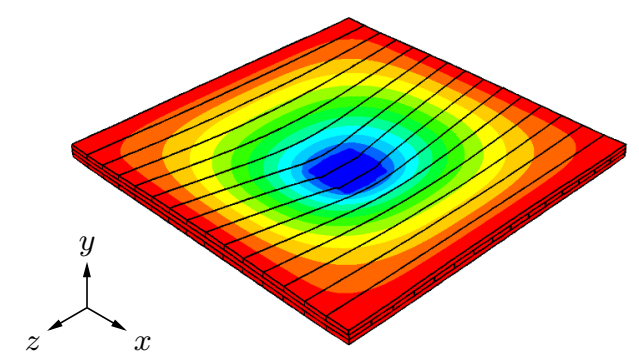

(a)

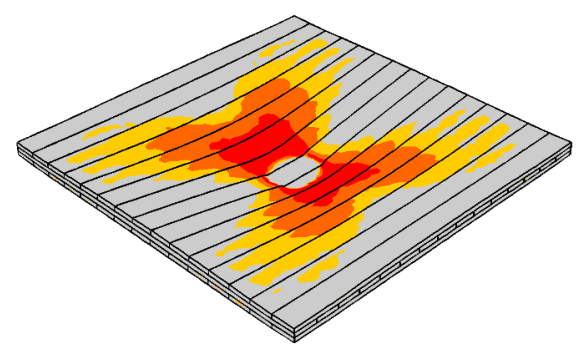

(b)

Figure 4: Illustrative example for numerical upper bound results using wooden boards from strength class C24 as: (a) the velocity field in the $y$ direction; (b) the failure mode with a threshold of $[-0.00024,-0.00016,-0.00008,0]$.

Beside the collapse load, a velocity field and an effective failure mode are obtained from each upper bound calculation, with illustrative examples for strength class C24, shown in Figures 4a and $4 \mathrm{~b}$, respectively. In the failure mode of Figure $4 \mathrm{~b}$, the velocity field at the point of structural failure is plotted on the deformed plate, and the failure region is marked by colours (from yellow to red) with red colours indicating the most critical failure.

The failure modes of CLT plates for different strength classes are compared in Figure 5, showing the loaded surface $\left(y^{+}\right.$surface in Figure $\left.4 \mathrm{a}\right)$. For all 3 strength classes, the plastic failure is initiated at the red region mainly due to kink-bending failure, and propagates sequentially through the orange and yellow regions due to a mixed mode of compressive and in-plane shear failures. In comparison, the failure region of the C18 plate is more localised, and in opposite, is more dispersed for the C35 plate. This agrees well with the experimental observation in Hochreiner et al. [27], that, at structural collapse, the failure region only covers $40 \%$ of the total plate width for $\mathrm{C} 18$ and $76 \%$ for C35. The reason for this behaviour is that, by using stronger wooden boards, higher tensile loads can be carried 


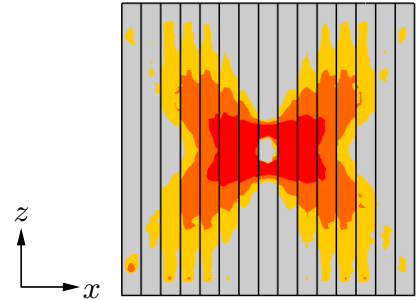

(a) $\mathrm{C} 18$

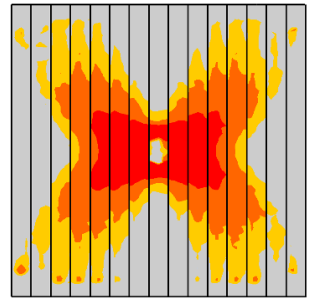

(b) $\mathrm{C} 24$

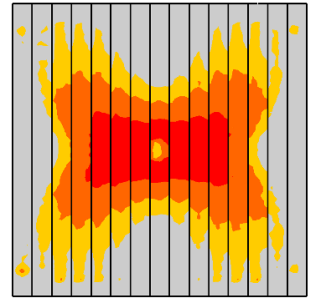

(c) C35

Figure 5: Comparison of upper bound failure modes observed on the loaded surface $\left(y^{+}\right.$surface in Figure 4a) for strength classes: (a) C18 with a threshold $[-0.00042,-0.00028,-0.00014,0]$; (b) C24 with a threshold $[-0.00024,-0.00016,-0.00008,0]$; (c) C35 with a threshold $[-0.0002,-0.00013,-0.00006,0]$.

in grain direction and the rolling shear strength is reached first in the middle layer close to the interface between other layers. Thus, the propagation of rolling shear failure in the middle layer leads to a stress redistribution and more wooden boards are activated to carry the bending load.
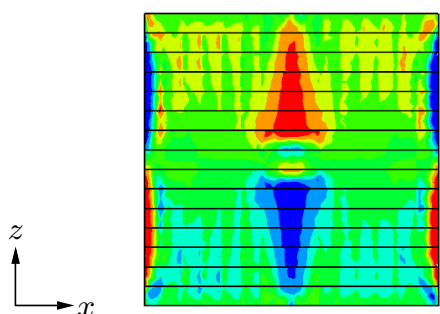

(a) $\mathrm{C} 18$
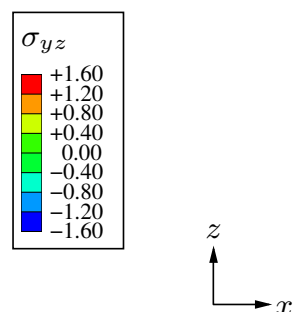

(b) $\mathrm{C} 35$
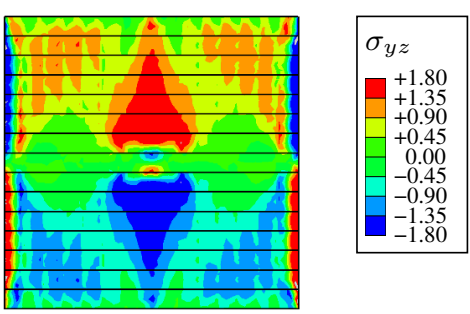

Figure 6: Comparison of the rolling shear stress fields in the middle layer of CLT plates for two different strength classes.

To verify this hypothesis, the shear stress fields $\sigma_{y z}$ on the interface between the middle layer and the top layer of CLT plates, considered as the rolling shear stress field for the middle layer, are compared in Figure 6 for $\mathrm{C} 18$ and C35. It can be noticed that high rolling shear stress regions cover larger areas of the surface of the CLT plate with stronger wooden boards, i.e. of the higher grading class, which agrees well with above-mentioned experimental observations and conclusions.

It is worth noting that a mesh of 32,868 tetrahedral elements, referred to as the initial mesh in the following, is used in each of the lower bound and upper bound limit analysis calculations. For a brief study on the mesh sensitivity, various discretisations of the CLT plate for C24 are considered with the number of elements varying from 32,868 to 73,744 . The corresponding lower and upper bound limit analysis results are illustrated in Figure 7. The bound gap decreases significantly from $7.59 \%$ for the coarsest mesh with 15,775 elements to $1.86 \%$ for the initial mesh. But further mesh refinement leads to only minor improvement in the quality of the numerical results, since the bound gap decreases from $1.86 \%$ to $1.03 \%$ when the number of elements is increased to 73,744 for the finest mesh. Thus, one can conclude that the initial mesh size chosen for the numerical calculations has been sufficiently fine to obtain good predictions on CLT strength behaviour.

Beside reliable estimates, the time efficiency of the numerical method is also remarkable. With a mesh of 40,000 tetrahedral elements, the total CPU time for each approximation is around $40 \mathrm{~min}$, including a lower bound and an upper bound calculation. Taking advantage of the high time efficiency, 


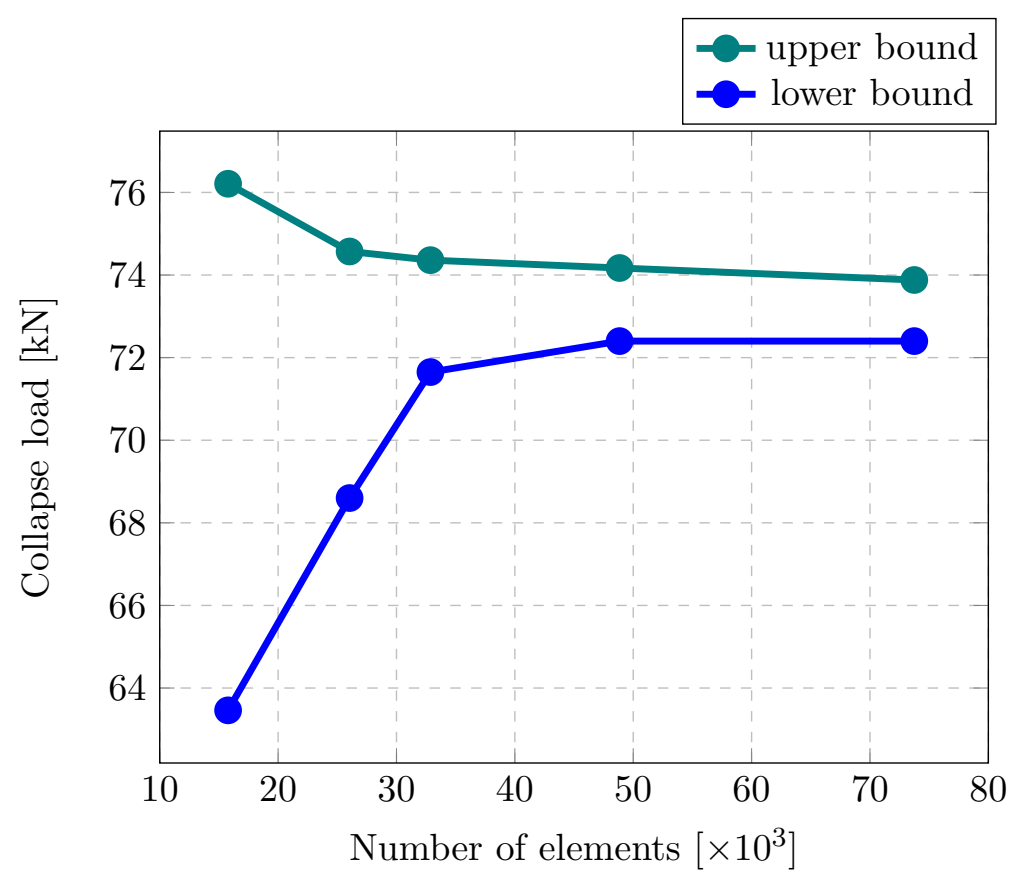

Figure 7: Mesh sensitivity study of the CLT plate bending test for strength class C24.

this numerical method provides the basis for a stochastic approach targeting a closer look into the failure mechanism of CLT plates with the consideration of property varieties and inhomogeneities of wooden boards by simulating a large number of models.

\subsection{Application using strength profiles}

To study the impact of inhomogeneities, in our case of knots, on the bending strength of CLT plates, we use a database of so-called strength profiles of real wooden boards. For this database, a total of 350 Norway spruce boards of strength grades LS15 (T14) and LS22 (T22) were, first, virtually reconstructed and, then, by using so-called indicating properties section-wise evaluated to obtain strength profiles of the longitudinal strength component for each board. The respective procedure is described in detail in [38] and summarised subsequently.

All boards were inspected using a tracheid effect-based laser scanning device, resulting in grid-like fibre orientation information available on all four board surfaces. In addition, photographs of the cross sections on both ends of the board allow for an estimation of pith locations. The fully automated approach described in [36] is used to obtain a geometrical description of the knot morphology. Therein, fibre angle measurements are used to estimate knot areas on the board's surfaces. This information in combination with the pith location is used to fit rotationally symmetric cones representing knots into the board. Here, the reconstruction error is minimised by employing a so-called simulatedannealing optimisation scheme, which reduces the difference between actual knot areas and knot areas obtained from intersecting the reconstructed cones with the board. Figure 8a-e shows the results for an exemplary board. In Figure 8c, all recognised knots are displayed, whereas in Figure 8d only the significant knot groups according to a modified version of the criterion presented in [37] are shown. Here, significance is defined by using a size measure for the knots. Knots with a visible knot area above the 70\%-quantile are considered to be large knots and, thus, to have a significant influence on the board's mechanical behaviour. In addition, the mutual distance between adjacent large knots is checked and those with longitudinal distances below $200 \mathrm{~mm}$ are grouped together. All 

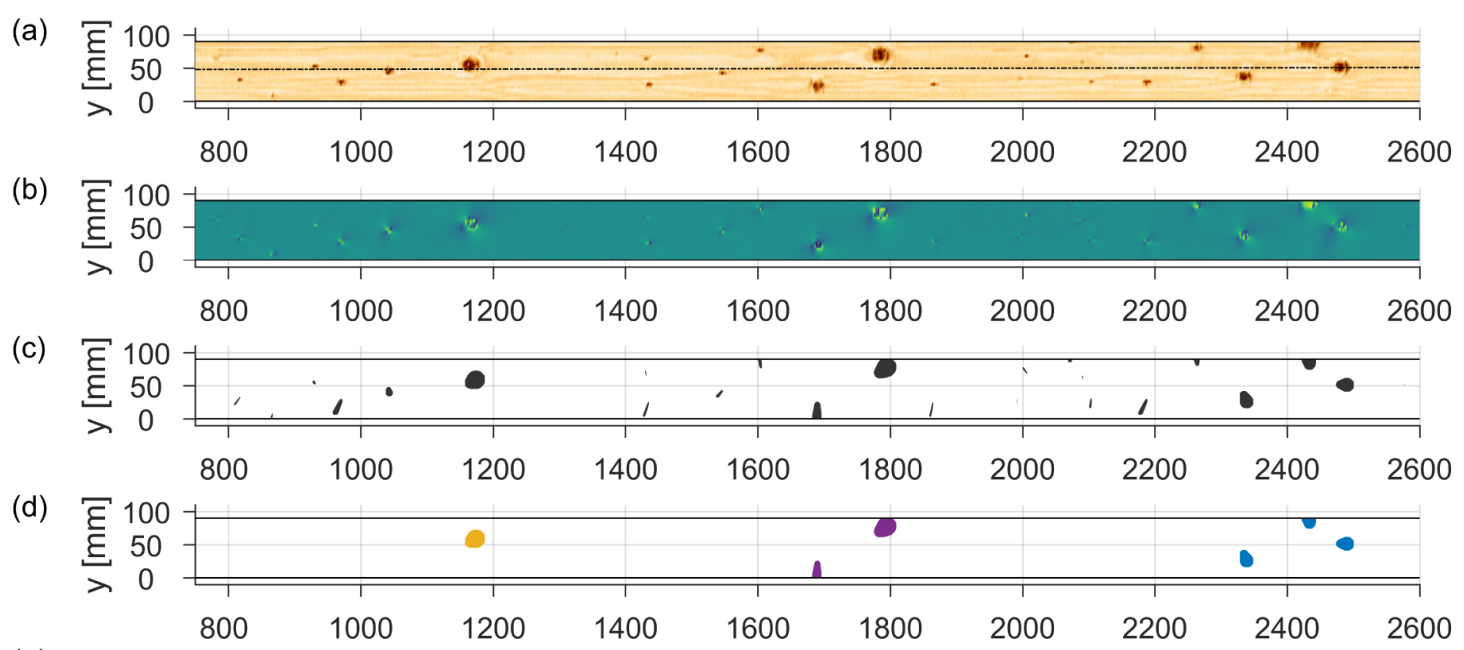

(e)
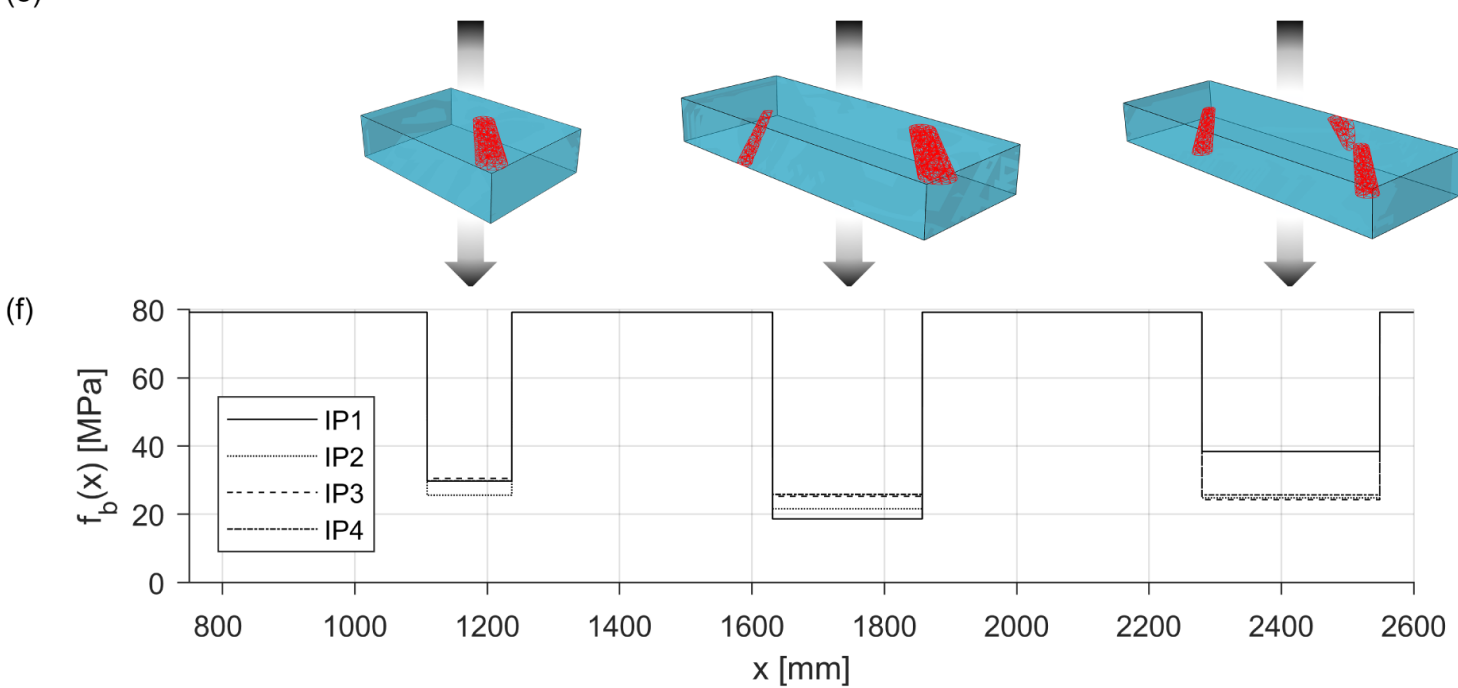

Figure 8: From board to strength profiles. (a) For a regular board, (b) based on laser scanning data (c) the knot fitting algorithm [36] automatically reconstructs the 3D knot geometry. (d) After the regrouping procedure has been applied, only the most important knot groups are retained in the model [37]. These knot groups, displayed in (e), are investigated using indicating properties (IPs). In (f), different strength profiles according to IPs 1 to 4 are displayed. (modified from [38]) 
other (small) knots are now disregarded unless they lie in close vicinity of larger knots, defined as a maximum distance of $100 \mathrm{~mm}$. The reason for this approach is that small knots are considered to be of small mechanical significance if by themselves, but they might have an influence on sections, where interacting multiple knots lead to variations in fibre deviations and, thus, influences on failure mechanisms. The board is now divided into so-called knot sections, with maximum lengths of $300 \mathrm{~mm}$, and interjacent clear-wood sections without knots. Each knot section is then subjected to a set of indicating properties, which are based on a study presented in [52] and are combinations of the following parameters:

- knot area ratio (KAR), which is the ratio of the projected knot areas to the cross-section area,

- knot area, which refers to the visible knot areas on the board's surfaces,

- weighted knot area, further distinguishes between knot areas on top/bottom and left/right surfaces,

- knot volume,

- interface areas, between knots and surrounding clear-wood, and

- Foley area ratio (FAR), which, in analogy to the KAR value, describes the ratio of the projected fibre deviation area to the cross section area.

These parameters were combined to a total of four indicating properties:

1. IP1: KAR, weighted knot area, knot volume, interface areas, FAR

2. IP2: KAR, knot area, knot volume, interface

\section{IP3: KAR}

4. IP4: KAR, knot area, knot volume

For the clear-wood sections, the longitudinal tensile strength is computed following the approach in [24], which is based on scaling experimentally obtained strength values according to density-dependent results obtained by a micromechanical model for elastic behaviour. The resulting strength profiles for the exemplary wooden board can be seen in Figure 8f. Such profiles of alternating defect-free and knot sections are referred to as weak zone models and are in accordance to [20,31,32].

The database of strength profiles provides the basis for a stochastic study on how wooden board properties influence the overall CLT capacities. In the experiments [27], wooden boards were graded into the bending-based strength classes, C18, C24, and C35, defined in EN 338:2009 [19]; for the strength profile database, wooden boards were graded into the tensile-based strength classes, LS15 and LS22, defined in EN 14081-4:2009 [17]. As mentioned in [3], by comparing the tensile strengths, the strength classes LS15 and LS22 can be considered as equivalent to C24 and C35, respectively. Thus, the application of numerical limit analysis using existing strength profiles to obtain CLT bending capacity estimates can also be validated using the experimental results for C24 and C35 shown in Figure 3.

In this work, the strength profile group IP4 is used in the calculations, as it has been proven to be reliable for strength estimates in [38]. From the strength profile database, both densities and longitudinal tensile strengths are available for knot sections and clear wood sections. In order to define the Tsai-Wu failure surfaces, all strength parameters have to be determined as shown in Eq. (23) which requires more strength components beside the longitudinal tensile strengths. For each clear wood section, all 9 strength components $\left(f_{t, L}, f_{t, R}, f_{t, T}, f_{c, L}, f_{c, R}, f_{c, T}, f_{\tau, L R}, f_{\tau, R T}\right.$ and $\left.f_{\tau, L T}\right)$ are determined by its density according to the strength-density correlation discussed in [24]. For knot 
sections, no reliable data for strength properties is available due to the lack of knowledge about failure mechanisms in the vicinity of knots, thus, in this work, the strength components are determined by the longitudinal tensile strengths using similar correlations as applied for the clear wood sections.

For each grading class, data for a total of 140 individual boards was available. During the assembly of the CLT plate models, for each simulation run, unique boards were randomly chosen from the database according to the studied grading class. The single boards were then divided into several knot and clear-wood sections with assigned strength parameters determined by the board's strength profile information. By re-running the random board selection process, for both strength classes, a big number of different CLT plates can be assembled using wooden boards with different strength profiles, and their failure behaviour under bending can be predicted using numerical limit analysis.

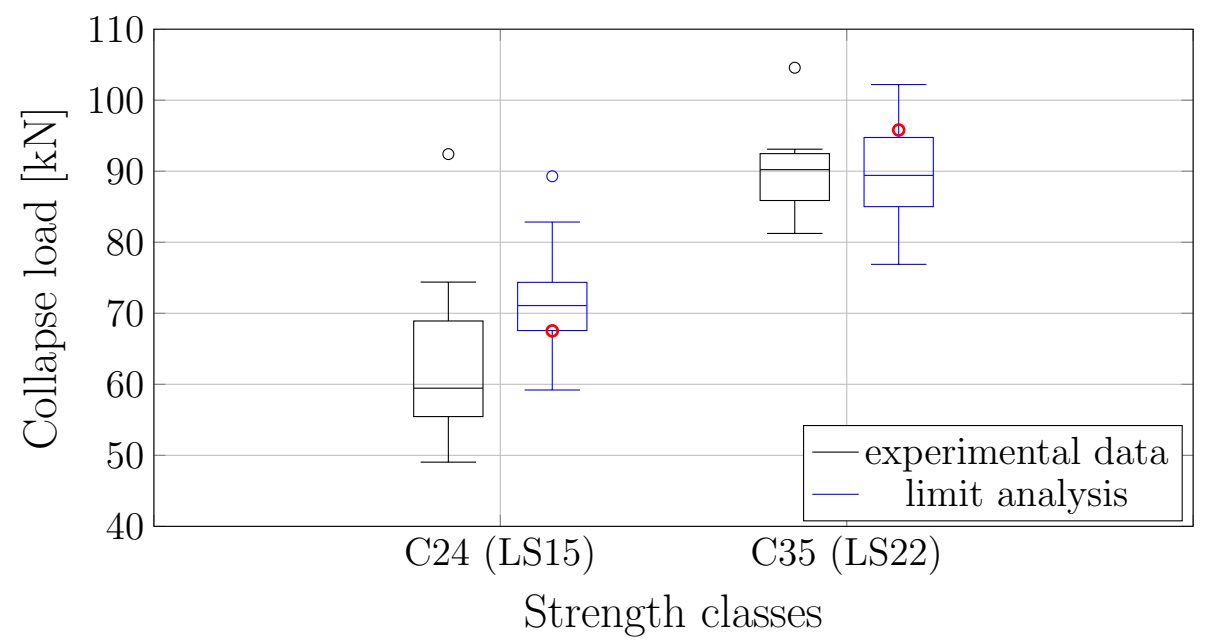

Figure 9: Comparison of numerical and experimental collapse loads of CLT plates under bending load using wooden boards from different strength classes (the red marked points denote the results of the exemplary CLT boards of Figures 11 and 13, and the black and blue marked points are outliers, i.e. values greater than upper quartile plus 1.5 times the interquartile range).

\begin{tabular}{|c|c|c|c|c|c|c|}
\hline \multirow{2}{*}{ type } & \multicolumn{3}{|c|}{ C24(LS15) } & \multicolumn{3}{c|}{ C35(LS22) } \\
\cline { 2 - 7 } & $\bar{x}$ & $s$ & $C O V$ & $\bar{x}$ & $s$ & $C O V$ \\
\hline experiment & 63.64 & 13.82 & 0.2172 & 90.37 & 6.93 & 0.0767 \\
\hline limit analysis & 71.17 & 6.02 & 0.0846 & 89.59 & 6.21 & 0.0693 \\
\hline
\end{tabular}

Table 2: Resulting mean value $\bar{x}[\mathrm{kN}]$, standard deviation $s[\mathrm{kN}]$ and coefficient of variation $C O V$ for both experimental and numerical results corresponding to different strength classes.

For each of the two strength classes, LS15 and LS22, 50 CLT plate models were generated. The collapse loads of all CLT plates from numerical limit analysis calculations are plotted in Figure 9 compared with the experimental results for strength classes C24 and C35. For strength class C35, the scatter for numerically-obtained collapse loads matches well to the experimental data points and the mean value $\bar{x}$ as well as the standard deviation $s$ for both numerical and experimental results agree well to each other shown in Table 2, indicating that the numerical estimates are reliable for this strength class in a stochastic manner. For the strength class C24, the scatter for the numerical results covers about $75 \%$ of the distribution of experiment data points in the higher end with higher mean value $\bar{x}$ for the numerical estimates. For an explanation it can be referred to the discussion in Section 3.1, that the numerical limit analysis is able to predict the ductile failure mechanisms of the 
CLT plates, as the dominant failure for C35, but leads to an overestimation of collapse loads when brittle failure mechanisms cannot be ignored, as for C24. Since failure mechanisms of CLT plates for C24 can be considered as a mixed mode of ductile and brittle responses, in Table 2, the standard deviation $s$ is higher for experimental results than for numerical results where only ductile response is considered.

Assessing all failure modes from the comprehensive numerical upper bound limit analysis calculations for both strength classes, basically two categories of failure modes are identified and the impact of wooden board strength profiles is discussed in the following.

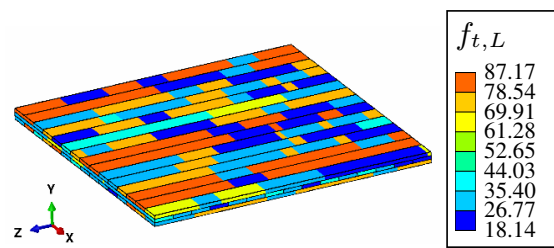

(a)

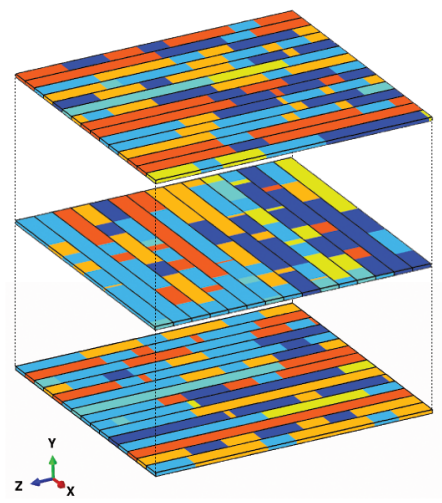

(c)

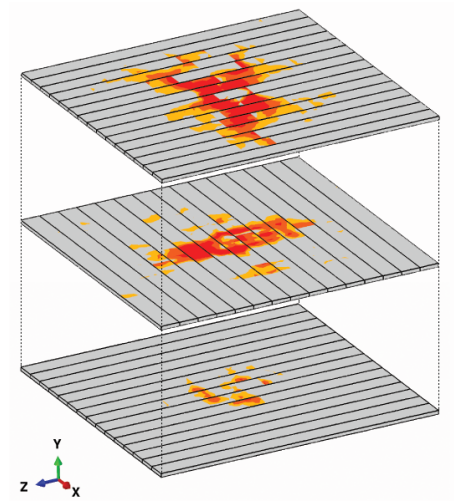

(d) Top view

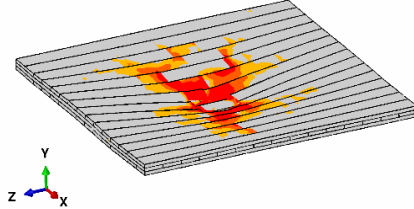

(b)

Figure 10: Selected upper bound results for the localised failure mode plotted as: (a) the longitudinal tensile strength profiles assigned in the 3-layer CLT plate; (b) the failure mode of this plat as the coloured region and the velocity field as the deformation; (c) the exploded view for strength profiles; (d) the exploded view for the failure mode from top; (e) the exploded view for the failure mode from bottom.

The first category is identified mainly in CLT plates for strength class C24, for which a selected example from one upper bound calculation is illustrated in Figure 10. The strength profiles, indicated by the longitudinal tensile strength, are illustrated for the whole plate in Figure 10a and for each layer in the exploded view Figure 10c, where weaker clear wood sections and a higher number of knot sections are present. The failure mode of the plate is plotted in Figure 10b, and, in order to have a complete image of failure pattern over the whole plate, exploded views from top and bottom are shown in Figure 10d and Figure 10e, respectively. It is observed that the dominant failure locates in the top layer, especially on the top surface (the loaded surface). The selected failure mode on the top surface is plotted in Figure 11a, where a localised plastic failure mechanism is observed. When the loading is applied, plastic failure is initiated due to the local instabilities around the loaded region (marked by the blue square) known as the kink-bending failure on the top wooden board layer as shown in the sectional view (1). Then the failure propagates through wooden boards perpendicularly mainly due to the longitudinal compressive failure, as shown in the sectional view (2), leading to the final structural collapse. In order to discuss the impact of wooden board inhomogeneities on the failure mode, the longitudinal tensile strength profiles are plotted and the failure mechanism identified in Figure 11a 


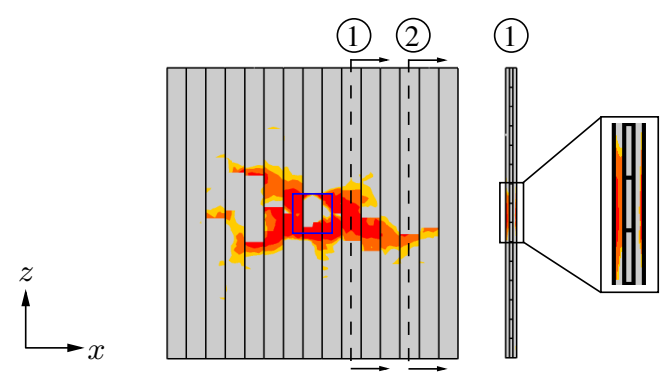

(a)

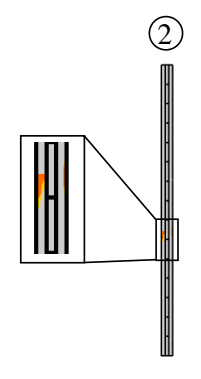

(2)

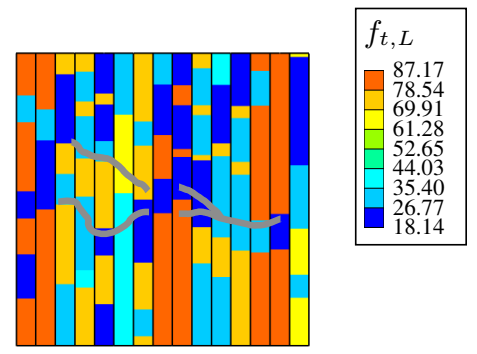

(b)

Figure 11: Illustration of the identified dominant failure mechanisms from the selected localised failure mode as: (a) the failure mode observed on the loaded surface (blue square indicates loaded region) as well as 2 view cuts (1) and (2) with zone-in views for the kink-bending failure and the longitudinal compressive failure, respectively; (b) the longitudinal tensile strength profiles assigned to wooden boards in the upper layer and the identified failure pattern (grey curves) according to the failure mode.

are indicated by grey curves in Figure 11b. It is obvious that, after the kink-bending failure initiated around the loaded region, the longitudinal compressive failure always propagates through wooden boards in the weak zones, normally knot sections, without plastic failure in surrounding clear wood sections. In this case, the failure mechanisms are mainly determined by the distributions of the knot sections leading to an insufficient utilisation of the strength properties of clear wood sections since they are not activated to carry loads during structural collapse.

The second category of failure modes is identified in CLT plates with stronger wooden boards, in this case from strength class C35, for which strength profiles of a selected example are plotted for the whole plate in Figure 12a and for each layer in Figure 12c. Since less knot sections are present, the localised failure cannot propagate through the whole plate and dispersed failure mechanisms are observed as shown in Figure $12 \mathrm{~b}$ as well as in the exploded views Figure 12d and Figure 12e, where the dominant failure is still observed on the top surface shown in Figure 13a. Compared to the first category, the main difference is that, instead of the localised failure region perpendicular to the wooden board orientation, the dispersed failure mechanism is observed in this case with the tip of the failure region aligning parallel to the wooden boards. This difference is attributed to the rolling shear failure in the middle layer prior to the structural collapse, shown in the view cut (1) and (2), which results in the stress redistribution in the top layer. Plotting failure patterns, identified in Figure 13a as grey curves in the strength profiles Figure 13b, the failure mechanisms of the CLT plate can be summarised in sequence as: 1) the plastic failure is initiated due to the kink-bending failure and propagates through the knot sections of wooden boards; 2) further propagation of the localised longitudinal compressive failure is interrupted due to the presence of strong clear wood sections; 3) the rolling shear failure occurs in the middle layer; 4) the strong wooden boards are activated to carry the load whose bending failure leads to the structural collapse.

The dominant failure mechanisms in above numerically-obtained failure modes are the longitudinal compressive failure, for the first category, and the rolling shear failure as well as the bending failure, for the second category, which agrees to the experimental observations in [26]. Additionally, similar conclusions can be drawn as mentioned in the experimental study of [27] that, by using stronger wooden boards in CLT plates, the increase in bending capacity is achieved not only because of the higher strength properties of wooden boards, but, more importantly, related to a more efficient utilisation, as more wooden boards are involved to carry the load. 


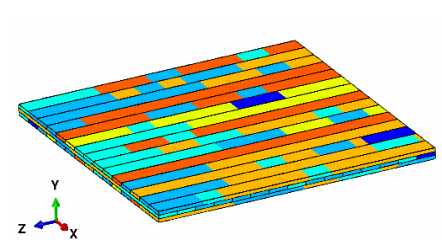

(a)

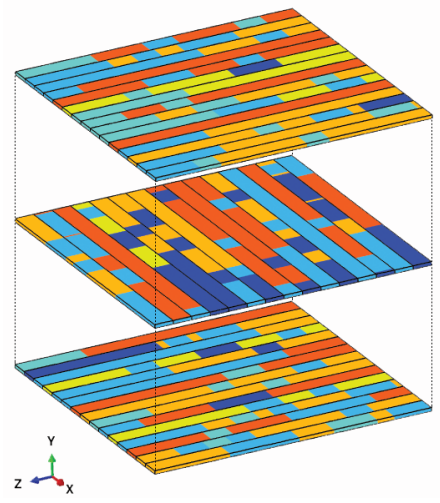

(c)
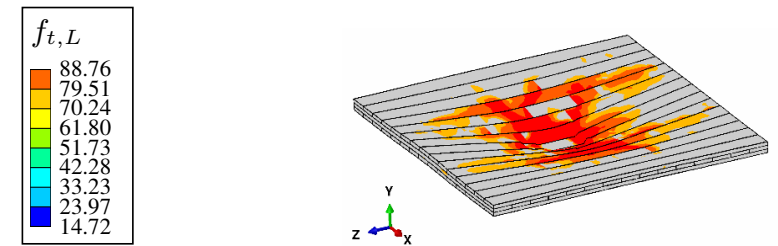

(b)

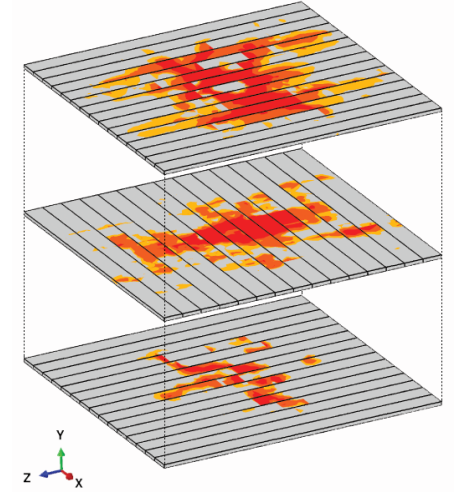

(d) Top view

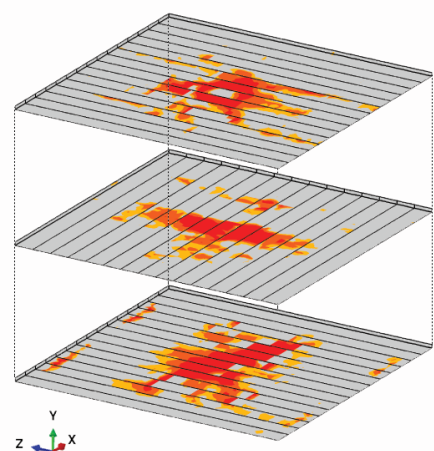

(e) Bottom view

Figure 12: Selected upper bound results for the dispersed failure mode plotted as: (a) the longitudinal tensile strength profiles assigned in the 3-layer CLT plate; (b) the failure mode of this plat as the coloured region and the velocity field as the deformation; (c) the exploded view for strength profiles; (d) the exploded view for the failure mode from top; (e) the exploded view for the failure mode from bottom.

\section{Summary and conclusions}

In this paper, 3D numerical limit analysis formulations were implemented for strength estimates of CLT plates for which, to the authors' knowledge, no sophisticated numerical method is available in timber engineering. In this method, the anisotropic Tsai-Wu failure criterion was employed to measure the material failure behaviour in wooden boards with respect to the local principal material orientations, and the strength parameters were determined from either code values in Eurocode 5 or a strength profile database. By using code values, the main focus was laid on the validation of the numerical method using experimental results and the influence of wooden board strengths on the CLT plate bending capacities; by using strength profiles, the main focus was laid on the impact of section-wise varying strength values, caused by inhomogeneities like knots, on the CLT plate failure mechanisms. Based on the obtained results, the main finding of this work can be summarised as:

- A validation of the numerical limit analysis approaches has been introduced using strength values from Eurocode 5 for 3 different strength classes, C18, C24, and C35, as input parameters, and good agreement between numerical and experimental results are observed. The dominant advantages of this numerical method rely on the small computational error, indicated by the small bound gap, and the high time efficiency over the conventional finite element method.

- By using a strength profile database, CLT plates are assembled using wooden boards with arbitrarily-assigned strength profiles, resulting in section-wise varying numerical strength estimates. Performing comprehensive calculations, the numerical upper bound calculations are able to provide reliable strength estimates with similar scatter as found in experimental results for strength classes C24 and C35. Distinct failure modes are obtained for each calculation with arbitrary combinations of strength profiles. 


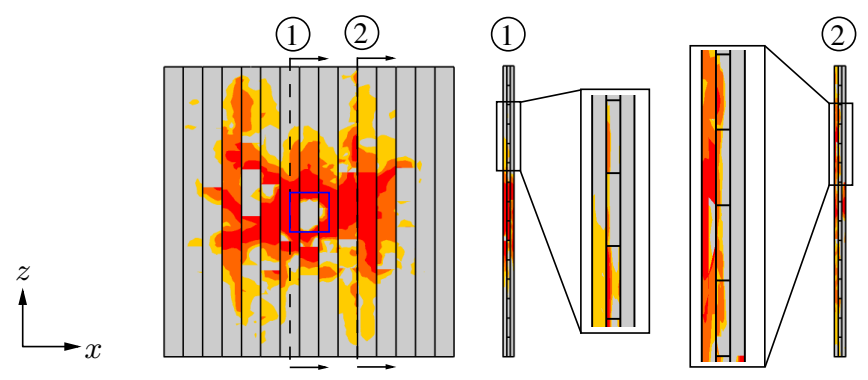

(a)
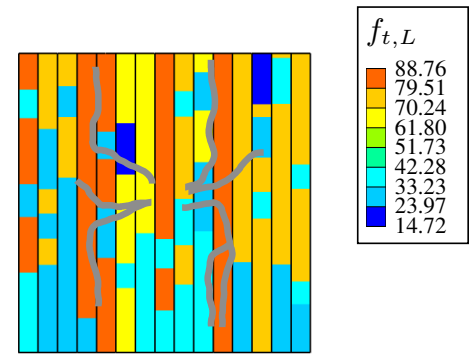

(b)

Figure 13: Illustration of the identified dominant failure mechanisms from the selected dispersed failure mode as: (a) the failure mode observed on the loaded surface (blue square indicates loaded region) as well as 2 view cuts (1) and (2) with zone-in views for the kink-bending failure and the longitudinal compressive failure, respectively; (b) the longitudinal tensile strength profiles assigned to wooden boards in the upper layer and the identified failure pattern (red curves) according to the failure mode.

- Due to the high time efficiency of this numerical method, the influence from section-wise varying strength values, caused by inhomogeneities in wooden boards, on the overall CLT plate capacities can be studied in a stochastic approach using a strength profile database. The numericallyobtained failure modes agree well with experimental observations that, 1) when weaker wooden boards (e.g. C24) are used, the failure mechanisms are more localised and propagate through weak links of wooden boards (normally knot sections) and both brittle and ductile responses can be observed at the point of structural collapse; 2) when stronger wooden boards (e.g. C35) are used, the rolling shear failure in the middle layer plays an important role and more wooden boards are activated to carry the bending load and the structural collapse is dominated by ductile response.

- For higher strength classes limit analysis is expected to deliver much more reliable results, since structural failure is mainly dominated by rolling shear failure, a failure mode which is characterised by almost ideal plastic behaviour in the associated material points and, thus, fulfilling the assumptions of limit analysis best. For lower strength classes, other failure modes, associated with strong softening behaviour in certain material points, become significant. A solution to that could be the application of a sequential limit analysis approach, which allows for modelling softening behaviour in a stable and efficient way. However, in this case the assumption of rigidity would be necessary to include, and only reliable results can be expected if there is no significant elastic-plastic coupling [41]. For structural brittle failure modes of wood products this cannot be assumed, however, for structural ductile behaviour, as often occurring in timber connections or in reinforced wood products, sequential limit analysis could be an appropriate method.

Accordingly, one can conclude that, the presented numerical limit analysis is a useful tool to fill the gap in timber engineering for efficient and reliable estimates on CLT plate bending capacities and structural failure mechanisms in which inhomogeneities and strength variants in wooden boards can be considered in a stochastic approach.

Future work is devoted to more sophisticated stochastic studies on the impact of inhomogeneities on the overall strength of CLT plates and the corresponding failure modes. The results are expected to provide more information about CLT plate failure mechanisms and serve as reference for the ongoing development of the wooden board grading process as well as the standardisation of CLT products. 
Additionally, for further study on the strength profiles, the presented numerical method will be applied for strength estimates of knot sections to predict failure mechanisms in vicinities of knots and surrounding fibre deviations.

Finally, it should be noted that an appropriate 2D limit analysis formulation for plate-like structures could lead to a further increase of computational efficiency, and would make limit analysis an even more attractive method for analysing collapse mechanisms in CLT structures. However, although there exist very promising yield design formulations for thick plates $[6,68,77]$, shells [5], as well as reinforced concrete [12], these formulations haven't been extended to general orthotropic strength behaviour and multilayered configurations yet. To accomplish that, the plasticity theories for composite laminated plates in [4] could serve as basis.

\section{Acknowledgements}

Financial support for this work in the framework of the PhDSchool DokInHolz funded by the Austrian Federal Ministry of Science, Research and Economy and the Austrian Association of Wood Industries is gratefully acknowledged. We also gratefully acknowledge the financial support of this work by the Austrian Science Fund (FWF) through the Erwin Schrödinger Fellowship J3748-N30.

\section{References}

[1] Aicher, S., Gustafsson, P. J., Haller, P., and Petersson, H. Fracture mechanics models for strength analysis of timber beams with a hole or a notch - A report of RILEM TC-133. Tech. rep., Structural Mechanics, 2002.

[2] Anderheggen, E., And Knöpfel, H. Finite element limit analysis using linear programming. International Journal of Solids and Structures 8, 12 (1972), 1413-1431.

[3] Bacher, M., And Krzosek, S. Bending and tension strength classes in European standards. Forestry and Wood Technology 88 (2014), 14-22.

[4] Bank, L. C., And Bieniek, M. P. Stress-resultant plasticity theories for composite laminated plates. International journal of plasticity 4, 4 (1988), 317-333.

[5] Bleyer, J., And De Buhan, P. A numerical approach to the yield strength of shell structures. European Journal of Mechanics-A/Solids 59 (2016), 178-194.

[6] Bleyer, J., Van Le, C., And De Buhan, P. Lockingfree discontinuous finite elements for the upper bound yield design of thick plates. International Journal for Numerical Methods in Engineering 103, 12 (2015), 894-913.

[7] Brandner, R., Flatscher, G., Ringhofer, A., Schickhofer, G., And Thiel, A. Cross laminated timber (CLT): overview and development. European Journal of Wood and Wood Products 74 (2016), 331-351.

[8] Capsoni, A., Corradi, L., And Vena, P. Limit analysis of anisotropic structures based on the kinematic theorem. International Journal of Plasticity 17, 11 (2001), 1531-1549.

[9] Capsoni, A., Corradi, L., and Vena, P. Limit analysis of orthotropic structures based on hill's yield condition. International Journal of Solids and Structures 38, 22-23 (2001), 3945-3963.

[10] Ciria, H., and Peraire, J. Computation of upper and lower bounds in limit analysis using second-order cone programming and mesh adaptivity. In 9th ASCE Specialty Conference on Probabilistic Mechanics and Structural Reliability (2004). 
[11] Ciria, H., Peraire, J., And Bonet, J. Mesh adaptive computation of upper and lower bounds in limit analysis. International Journal for Numerical Methods in Engineering 75, 8 (2008), 899944.

[12] Domenico, D. D., Pisano, A., And Fuschi, P. A FE-based limit analysis approach for concrete elements reinforced with FRP bars. Composite Structures 107 (2014), 594-603.

[13] Dorn, M. Investigations on the serviceability limit state of dowel-type timber connections. $\mathrm{PhD}$ thesis, Vienna University of Technology, 2012.

[14] Drucker, D. C., Greenberg, H. J., and Prager, W. The safety factor of an elastic-plastic body in plane strain. Journal of Applied Mechanics 18 (1951), 371-378.

[15] Drucker, D. C., Prager, W., and Greenberg, H. J. Extended limit design theorems for continuous media. Quarterly of Applied Mathematics 9 (1952), 381-389.

[16] Eberhardsteiner, J. Experimentelle Bestimmung der biaxialen Festigkeitseigenschaften (in German). Springer-Verlag Wien, 2002.

[17] EN 14081-4. Timber structures - Strength graded structural timber with rectangular cross section - Part 4: Machine Grading - Grading machine settings for machine controlled systems. European Committee for Standardization, Brussels (2009).

[18] EN 1995-1-1. Eurocode 5 - Design of timber structures - Part 1-1: General rules and rules for buildings. Tech. rep., Comité Européen de Normalisation. Bruxelles, Belgium, 2003.

[19] EN 338. Structural timber - Strength classes. European Committee for Standardization, Brussels (2009).

[20] Fink, G., Frangi, A., And Kohler, J. Bending tests on GLT beams having well-known local material properties. Materials and Structures 48, 11 (2015), 3571-3584.

[21] Füssl, J., Bader, T., and Eberhardsteiner, J. Computational mechanics for advanced timber engineering - from material modeling to structural applications. IACM Expressions 32 (2012), 6-11.

[22] Füssl, J., Li, M., Lukacevic, M., Eberhardsteiner, J., and Martin, C. M. Comparison of unit cell-based computational methods for predicting the strength of wood. Engineering Structures 141 (2017), 427-443.

[23] Gagnon, S., And Pirvu, C. CLT Handbook: cross-laminated timber, Canadian ed. FPInnovations, 2011.

[24] Hackspiel, C., De Borst, K., and Lukacevic, M. A numerical simulation tool for wood grading model development. Wood Science and Technology 48, 3 (2014), 633-649.

[25] Hill, R. On the state of stress in a plastic-rigid body at the yield point. Philosophical Magazine $42(1951), 868-875$.

[26] Hochreiner, G., Füssl, J., and Eberhardsteiner, J. Cross-laminated timber plates subjected to concentrated loading - Experimental identification of failure mechanisms. Strain 50, 1 (2013), 68-81.

[27] Hochreiner, G., Füssl, J., Serrano, E., and Eberhardsteiner, J. Influence of wooden board strength class on the performance of cross-laminated timber plates investigated by means of full-field deformation measurements. Strain 50, 2 (2014), 161-173. 
[28] Hristov, P., DiazDelaO, F., Flores, E. S., Guzmn, C., and Farooq, U. Probabilistic sensitivity analysis to understand the influence of micromechanical properties of wood on its macroscopic response. Composite Structures 181 (2017), 229-239.

[29] Jenkel, C., Leichsenring, F., Graf, W., and Kaliske, M. Stochastic modelling of uncertainty in timber engineering. Engineering Structures 99 (2015), 296-310.

[30] Johansson, C.-J. Grading of Timber with Respect to Mechanical Properties. Wiley, 2003, ch. 3, pp. $23-43$.

[31] Källsner, B., And Ditlevsen, O. Lengthwise bending strength variation of structural timber. In IUFRO S 5.02 Timber Engineering (1994), Sydney, Australia.

[32] Källsner, B., Ditlevsen, O., and Salmela, K. Experimental verification of a weak zone model for timber in bending. In IUFRO S 5.02 Timber Engineering (1997), vol. 5, pp. 18-20.

[33] Kandler, G., And Füssl, J. A probabilistic approach for the linear behaviour of glued laminated timber. Engineering Structures 148 (2017), 673-685.

[34] Kandler, G., Füssl, J., and Eberhardsteiner, J. Stochastic finite element approaches for wood-based products: theoretical framework and review of methods. Wood Science and Technology 49, 5 (2015), 1055-1097.

[35] Kandler, G., Füssl, J., Serrano, E., And Eberhardsteiner, J. Effective stiffness prediction of GLT beams based on stiffness distributions of individual lamellas. Wood Science and Technology 49, 6 (2015), 1101-1121.

[36] Kandler, G., Lukacevic, M., And Füssl, J. An algorithm for the geometric reconstruction of knots within timber boards based on fibre angle measurements. Construction and Building Materials 124 (2016), 945-960.

[37] Kandler, G., Lukacevic, M., and Füssl, J. From the knot morphology of individual timber boards to the mechanical properties of glued laminated timber. In WCTE 2016 - World Conference on Timber Engineering (2016).

[38] Kandler, G., Lukacevic, M., Füssl, J., Zechmeister, C., And Wolff, S. Stochastic engineering framework for timber structural elements and its application to glued laminated timber beams. Construction and Building Materials 190 (2017), 573-592.

[39] Krabbenhøft, K., And Damkilde, L. Limit Analysis Based On Lower-Bound Solutions And Nonlinear Yield Criteria. Civil-Comp Press, 2000, pp. 117-129.

[40] Krabbenhøft, K., Lyamin, A., and Sloan, S. Formulation and solution of some plasticity problems as conic programs. International Journal of Solids and Structures 44, 5 (2007), 15331549 .

[41] Leblond, J.-B., Kondo, D., Morin, L., And Remmal, A. Classical and sequential limit analysis revisited. Comptes Rendus Mecanique 346, 4 (2018), 336-349.

[42] LI, H. Limit analysis of composite materials with anisotropic microstructures: A homogenization approach. Mechanics of Materials 43, 10 (2011), 574-585.

[43] LI, H. Microscopic limit analysis of cohesive-frictional ccomposite with non-associated plastic flow. European Journal of Mechanics A/Solids 37 (2013), 281-293. 
[44] Li, M., Füssl, J., Lukacevic, M., Eberhardsteiner, J., and Martin, C. M. An algorithm for adaptive introduction and arrangement of velocity discontinuities within 3d finite-elementbased upper bound limit analysis approaches. Computer Methods in Applied Mechanics and Engineering (Dec. 2018).

[45] Li, M., Füssl, J., Lukacevic, M., Eberhardsteiner, J., and Martin, C. M. A numerical upper bound formulation with sensible-arranged velocity discontinuities and orthotropic material strength behaviour. Journal of Theoretical and Applied Mechanics 56, 2 (2018), 417-433.

[46] Li, M., Füssl, J., Lukacevic, M., Eberhardsteiner, J., and Martin, C. M. Strength predictions of clear wood at multiple scales using numerical limit analysis approaches. Computers E Structures 196 (2018), 200-216.

[47] Limam, O., Foret, G., And Ehrlacher, A. RC beams strengthened with composite material: a limit analysis approach and experimental study. Composite Structures 59, 4 (2003), 467-472.

[48] Limam, O., Foret, G., And Ehrlacher, A. RC two-way slabs strengthened with CFRP strips: experimental study and a limit analysis approach. Composite Structures 60, 4 (2003), $467-471$.

[49] Limam, O., Foret, G., And Zenzri, H. Ultimate strength of pin-loaded composite laminates: A limit analysis approach. Composite Structures 93, 4 (2011), 1217-1224.

[50] Lukacevic, M., AND Füsst, J. Numerical simulation tool for wooden boards with a physically based approach to identify structural failure. European Journal of Wood and Wood Products 72, 4 (2014), 497-508.

[51] Lukacevic, M., AND Füssl, J. Application of a multisurface discrete crack model for clear wood taking into account the inherent microstructural characteristics of wood cells. Holzforschung 70,9 (2016), 845-853.

[52] Lukacevic, M., Füssl, J., and Eberhardsteiner, J. Discussion of common and new indicating properties for the strength grading of wooden boards. Wood Science and Technology 49, 3 (2015), 551-576.

[53] Lukacevic, M., Füssl, J., And Lampert, R. Failure mechanisms of clear wood identified at wood cell level by an approach based on the extended finite element method. Engineering Fracture Mechanics 144 (2015), 158-175.

[54] Lukacevic, M., Lederer, W., And Füssl, J. A microstructure-based multisurface failure criterion for the description of brittle and ductile failure mechanisms of clear-wood. Engineering Fracture Mechanics 176 (2017), 83-99.

[55] Lyamin, A., And Sloan, S. Upper Bound Limit Analysis using Linear Finite Elements and Nonlinear Programming. Civil-Comp Press, 2000, pp. 131-145.

[56] Lyamin, A. V., ANd Sloan, S. W. Lower bound limit analysis using non-linear programming. International Journal for Numerical Methods in Engineering 55, 5 (October 2002), 573-611.

[57] Lyamin, A. V., And Sloan, S. W. Upper bound limit analysis using linear finite elements and non-linear programming. International Journal for Numerical and Analytical Methods in Geomechanics2 26, 2 (2002), 181-216.

[58] LYSMER, J. Limit analysis of plane problems in soil mechanics. Journal of the Soil Mechanics and Foundations Division 96, 4 (1970), 1311-1334. 
[59] Mackenzie-Helnwein, P., Eberhardsteiner, J., And Mang, H. A. A multi-surface plasticity model for clear wood and its application to the finite element analysis of structural details. Computational Mechanics 31, 1-2 (2003), 204-218.

[60] Maier, G., Zavelani-Rossi, A., and Benedetti, D. A finite element approach to optimal design of plastic structures in plane stress. International Journal for Numerical Methods in Engineering 4, 4 (1972), 455-473.

[61] Makrodimopoulos, A. Remarks on some properties of conic yield restrictions in limit analysis. International Journal for Numerical Methods in Biomedical Engineering 26, 11 (2010), 1449-1461.

[62] Makrodimopoulos, A., And Martin, C. M. Lower bound limit analysis of cohesive-frictional materials using second-order cone programming. International Journal for Numerical Methods in Engineering 66, 4 (2006), 604-634.

[63] Makrodimopoulos, A., And Martin, C. M. Upper bound limit analysis using simplex strain elements and second-order cone programming. International Journal for Numerical and Analytical Methods in Geomechanics 31, 6 (2007), 835-865.

[64] Masuda, M. Theoretical consideration on fracture criteria of wood - proposal of finite small area theory. In Proceedings of the 1988 International Conference on Timber Engineering (Seattle, 1988), vol. 2, pp. 584-595.

[65] Milani, G. FE homogenized limit analysis model for masonry strengthened by near surface bed joint FRP bars. Composite Structures 92, 2 (2010), 330-338.

[66] Milani, G., And Bucchi, A. Kinematic FE homogenized limit analysis model for masonry curved structures strengthened by near surface mounted FRP bars. Composite Structures 93, 1 (2010), 239-258.

[67] Milani, G., Milani, E., And Tralli, A. Approximate limit analysis of full scale FRPreinforced masonry buildings through a 3D homogenized FE package. Composite Structures 92, 4 (2010), 918-935.

[68] Nguyen-Thoi, T., Phung-Van, P., Nguyen-Thoi, M. H., and Dang-Trung, H. An upperbound limit analysis of mindlin plates using cs-dsg3 method and second-order cone programming. Journal of Computational and Applied Mathematics 281 (2015), 32-48.

[69] Pech, S., Kandler, G., Lukacevic, M., And Füssl, J. Metamodel assisted optimization of glued laminated timber beams by using metaheuristic algorithms. Engineering Applications of Artificial Intelligence 79 (2019), 129-141.

[70] Pisano, A., Fuschi, P., And Domenico, D. D. A layered limit analysis of pinned-joints composite laminates: Numerical versus experimental findings. Composites Part B: Engineering 43, 3 (2012), 940-952.

[71] Pisano, A., Fuschi, P., And Domenico, D. D. Peak load prediction of multi-pin joints FRP laminates by limit analysis. Composite Structures 96 (2013), 763-772.

[72] Pisano, A., Fuschi, P., And Domenico, D. D. Numerical limit analysis of steel-reinforced concrete walls and slabs. Computers $\& 3$ Structures 160 (2015), 42-55.

[73] Saavedra Flores, E., Saavedra, K., Hinojosa, J., Chandra, Y., and Das, R. Multiscale modelling of rolling shear failure in cross-laminated timber structures by homogenisation and cohesive zone models. International Journal of Solids and Structures 81 (2016), 219-232. 
[74] Saavedra Flores, E. I., Ajaj, R., Dayyani, I., Chandra, Y., and Das, R. Multi-scale model updating for the mechanical properties of cross-laminated timber. Computers \& Structures 177 (2016), 83-90.

[75] Saavedra Flores, E. I., Dayyani, I., Ajaj, R., Castro-Triguero, R., DiazDelaO, F., DAs, R., AND Soto, P. G. Analysis of cross-laminated timber by computational homogenisation and experimental validation. Composite Structures 121 (2015), 386-394.

[76] Salencon, J. Introduction to the yield design theory and its applications to soil mechanics. European Journal of Mechanics, A/Solids 9, 5 (1990), 477-500.

[77] Sanchez-Palencia, E., And Zaoui, A. Homogenization Techniques for Composite Media. Springer-Verlag Berlin ; New York, 1987.

[78] Schmidt, J., And Kaliske, M. Zur dreidimensionalen Materialmodellierung von Fichtenholz mittels eines Mehrflächen-Plastizitätsmodells (in German). Holz als Roh- und Werkstoff 64, 5 (2006), 393-402.

[79] Schmidt, J., AND Kaliske, M. Simulation of cracks in wood using a coupled material model for interface elements. Holzforschung 61, 4 (2007), 382-389.

[80] Schmidt, J., And Kaliske, M. Models for numerical failure analysis of wooden structures. Engineering Structures 31, 2 (2009), 571-579.

[81] Serrano, E., and Gustafsson, P. J. Fracture mechanics in timber engineering - Strength analyses of components and joints. Materials and Structures 40, 1 (2007), 87-96.

[82] SjöDin, J., And Serrano, E. A numerical study of methods to predict the capacity of multiple steel-timber dowel joints. Holz als Roh- und Werkstoff 66, 6 (2008), 447-454.

[83] Sjödin, J., Serrano, E., And Enquist, B. An experimental and numerical study of the effect of friction in single dowel joints. Holz als Roh- und Werkstoff 66, 5 (2008), 363-372. 